\title{
TOPOLOGICAL METHODS IN ABSTRACT ALGEBRA. COHOMOLOGY THEORY OF GROUPS ${ }^{1}$
}

\author{
SAMUEL EILENBERG
}

1. Introduction. The title of this article requires some explanation. The term "abstract algebra" was used to indicate that we shall deal with purely algebraic objects like groups, algebras and Lie algebras rather than topological groups, topological algebras, and so on. The method of study is also purely algebraic but is the replica of an algebraic process which has been widely used in topology, thus the words "toplogical methods" could be replaced by "algebraic methods suggested by algebraic topology." These purely algebraic theories do, however, have several applications in topology.

The algebraic process borrowed from topology is the following. Consider a sequence of abelian groups $\left\{C^{q}\right\}$ and homomorphisms $\delta$,

$$
C^{0} \stackrel{\delta}{\rightarrow} C^{1} \rightarrow \cdots \rightarrow C^{q-1} \stackrel{\delta}{\rightarrow} C^{q} \stackrel{\delta}{\rightarrow} C^{q+1} \rightarrow \cdots
$$

such that $\delta \delta=0$. In each group $C^{q}$ two subgroups are distinguished

$$
\begin{aligned}
& Z^{q}=\text { kernel of } \delta: C^{q} \rightarrow C^{q+1}, \\
& B^{q}=\text { image of } \delta: C^{q-1} \rightarrow C^{q}
\end{aligned}
$$

with the second definition completed by setting $B^{0}=0$. The condition $\delta \delta=0$ is then equivalent with $B^{q} \subset Z^{q}$. The group

$$
H^{q}=Z^{q} / B^{q}
$$

is called the $q$ th cohomology group of the sequence (1.1). The elements of the groups $C^{q}, Z^{q}, B^{q}$ and $H^{q}$ are called $q$-dimensional cochains, cocycles, coboundaries and cohomology classes respectively. Each cocycle $z \in Z^{q}$ determines a cohomology class as the coset $\{z\}=z+B^{q}$. Two cocycles in the same coset are called cohomologous.

The process just described can be applied in the following three instances.

I. Groups. Let $Q$ be a multiplicative group and $G$ an abelian group written additively. Assume further that $Q$ operates on $G$, that is, that

An address delivered before the Summer Meeting of the Society on September 15, 1945, by invitation of the Committee to Select Hour Speakers for Annual and Summer Meetings; received by the editors May 29, 1948.

1 The presentation has been brought up to date and includes many results obtained after 1945. 
for each $x \in Q$ and $g \in G$ an element $x g \in G$ is given such that

$$
x\left(g_{1}+g_{2}\right)=x g_{1}+x g_{2}, \quad x_{2}\left(x_{1} g\right)=\left(x_{2} x_{1}\right) g, \quad 1 g=g
$$

where 1 is the unit element in $Q$. The group $C^{q}(Q, G)$ of the $q$-dimensional cochains of $Q$ in $G$ is defined as the group of all functions $f$ of $q$ variables in $Q$ with values in $G$. Addition in $C^{q}(Q, G)$ is defined by

$$
\left(f_{1}+f_{2}\right)\left(x_{1}, \cdots, x_{q}\right)=f_{1}\left(x_{1}, \cdots, x_{q}\right)+f_{2}\left(x_{1}, \cdots, x_{q}\right) \text {. }
$$

The coboundary homomorphism $\delta: C^{q}(Q, G) \rightarrow C^{q+1}(Q, G)$ is defined by the formula

$$
\begin{aligned}
(\delta f)\left(x_{1}, \cdots, x_{q+1}\right)= & x_{1} f\left(x_{2}, \cdots, x_{q+1}\right) \\
& +\sum_{i=1}^{q}(-1)^{i} f\left(x_{1}, \cdots, x_{i} x_{i+1}, \cdots, x_{q+1}\right) \\
& +(-1)^{q+1} f\left(x_{1}, \cdots, x_{q}\right) .
\end{aligned}
$$

The relation $\delta \delta=0$ is verified by computation. The resulting cohomology group $H^{q}(Q, G)$ is called the $q$ th cohomology group of $Q$ over $G$. Note that the operators of $Q$ on $G$ are part of the definition and a change in these operators will generally alter the group $H^{q}(Q, G)$.

II. Associative algebras. Let $A$ be an associative algebra over a field $F$, and $V$ a vector space over the same field. Assume further that $V$ is a two sided $A$-module, that is, that for every $a \in A, v \in V$ elements $a v$ and $v a$ of $V$ are defined which are bilinear functions of $a$ and $v$, and satisfy

$$
a_{2}\left(a_{1} v\right)=\left(a_{2} a_{1}\right) v, \quad a_{2}\left(v a_{1}\right)=\left(a_{2} v\right) a_{1}, \quad\left(v a_{2}\right) a_{1}=v\left(a_{2} a_{1}\right) .
$$

The group $C^{q}(A, V)$ of the $q$-dimensional cochains of $A$ in $V$ is defined as the group of all functions $f$ of $q$ variables in $A$ with values in $V$, linear with respect to each variable. Addition in $C^{q}(A, V)$ is defined as before. The coboundary formula is

$$
\begin{aligned}
(\delta f)\left(a_{1}, \cdots, a_{q+1}\right)= & a_{1} f\left(a_{2}, \cdots, a_{q+1}\right) \\
& +\sum_{i=1}^{q}(-1)^{i} f\left(a_{1}, \cdots, a_{i} a_{i+1}, \cdots, a_{q+1}\right) \\
& +f\left(a_{1}, \cdots, a_{q}\right) a_{q+1} .
\end{aligned}
$$

The resulting cohomology group $H^{q}(A, V)$ is the $q$ th cohomology group of $A$ over $V$. The groups $C^{q}, \cdots, H^{q}$ in this case are clearly vector spaces over the field $F$.

III. Lie algebras. Let $L$ be a Lie algebra over a field $F$ and let $V$ 
be a vector space over $F$. Assume further that a representation $P$ of $L$ in $V$ is given, that is, that for every $x \in L$ a linear transformation $P_{x}: V \rightarrow V$ is given such that $P_{x}$ is a linear function of $x \in L$ and that

$$
P_{y} P_{x}-P_{x} P_{y}=P_{[x, y]}
$$

for $x, y \in L$. The group $C^{q}(L, P)$ is defined as the group of all functions $f$ of $q$ variables in $L$ with values in $V$, linear with respect to each variable and alternating with respect to permutations of the variables. Addition in $C^{q}(L, P)$ is defined as before. The coboundary formula is $(\delta f)\left(x_{1}, \cdots, x_{q+1}\right)$

$$
\begin{aligned}
= & \sum_{k<l}(-1)^{k+l+1} f\left(\left[x_{k}, x_{l}\right], x_{1}, \cdots, \hat{x}_{k}, \cdots, \hat{x}_{l}, \cdots, x_{q+1}\right) \\
& +\sum_{i=0}^{q+1}(-1)^{i+1} P_{x_{i}} f\left(x_{1}, \cdots, \hat{x}_{i}, \cdots, x_{q+1}\right),
\end{aligned}
$$

where the symbol under the circumflex is to be omitted. The resulting cohomology group $H^{q}(L, P)$ is called the $q$ th cohomology group of $L$ over $P$ and clearly is a vector space over $F$. In the special case when $V=F$ and the representation $P$ is trivial (that is, when $P_{x}=0$ for all $x \in L)$, the second part of the coboundary formula is zero. We then write $H^{q}(L)$ instead of $H^{q}(L, P)$.

In all the three cases discussed above the convention prevails that a function of zero variables with values in $G$ is an element of $G$. Thus $C^{0}(Q, G)=G, C^{0}(A, V)=V, C^{0}(L, P)=V$.

A certain common feature can be observed in all the points at which the cohomology theories defined above come into contact with established parts of algebra. The cocycles almost invariably arise as "deviations" from a certain simple behavior. This point is not sufficiently formalized to be discussed here, but will be emphasized as the various instances come up. A strong analogy with the "obstructions" considered in topology in problems on extension and classification of mappings and in the theory of fiber bundles is suggested. In some instances the "deviations" and the "obstructions" actually come into contact.

The definition of the cohomology groups of groups was given by the author and S. MacLane in 1943 ([12], [13], [15], see also Hopf [26] and Eckmann [6]). ${ }^{2}$ The remainder of this paper is devoted to the discussion of these groups. After some preliminaries (\$\$2-3) we describe (\$\$4-8) the connections that have been established between this cohomology theory and other parts of algebra. This is

${ }^{2}$ Numbers in brackets refer to the bibliography at the end of the paper. 
followed ( $\$ 9-13)$ by the description of some intrinsic properties of the cohomology groups; these are often helpful in actual computations. Some contacts with topology become apparent. Finally (\$\$14-17) we give an account of the topological applications of the cohomology groups.

The cohomology theory of associative algebras was studied by Hochschild [22], [23], [24]. As yet, there are no applications to topology. Some of the algebraic results are analogous to the corresponding theorems in cohomology theory of groups.

The cohomology groups of Lie algebras were studied by Chevalley and the author [5]. The contact with topology is established by the fact that the cohomology groups (real coefficients) of the space of a compact Lie group are isomorphic to the cohomology groups of its Lie algebra. The theory is still in a very rudimentary stage and needs further development.

2. Alternative definitions. If we compare the definition of the coboundary $\delta f$ for the cases of groups and associative algebras we observe that they are formally identical except for the right operator on the last term. The two formulae can be brought to coincidence by assuming that the group $Q$ acts on $G$ as a group of two-sided operators, that is, that in addition to the left operators of $Q$ on $G$, the group $Q$ operates on $G$ also on the right in such a way that

$$
\begin{gathered}
\left(g_{1}+g_{2}\right) x=g_{1} x+g_{2} x, \quad\left(g x_{1}\right) x_{2}=g\left(x_{1} x_{2}\right), \quad g 1=g, \\
\left(x_{1} g\right) x_{2}=x_{1}\left(g x_{2}\right) .
\end{gathered}
$$

The formula for $\delta f$ is then modified by replacing the last term $4 f\left(x_{1}, \cdots, x_{q}\right)$ by $f\left(x_{1}, \cdots, x_{q}\right) x_{q+1}$. The cohomology group $H^{q}(Q, G)$ thus obtained includes the earlier groups as special cases, namely when the right operators of $Q$ on $G$ are simple (that is, when $g x=g$ for all $x \in Q, g \in G)$. It turns out that this generalization is not essential. In fact given that $Q$ operates on $G$ two-sidedly, define the group $O G$ which coincides with $G$ as a group but has $Q$ as a group of left operators as follows

$$
x \circ g=x g x^{-1} .
$$

Define a map

$$
\phi: C^{q}(Q, \circ G) \rightarrow C^{q}(Q, G)
$$

by setting

$$
(\phi f)\left(x_{1}, \cdots, x_{q}\right)=f\left(x_{1}, \cdots, x_{q}\right) x_{1} \cdots x_{q} .
$$


The maps $\phi$ are isomorphisms and commute with the coboundary operators, thus inducing isomorphisms of the cohomology groups $H(Q, G)$ (with two-sided operators) and $H(Q, \circ G)$ (with left operators). From now on, unless the contrary is specified, we shall adhere to the original definition involving left operators only.

A cochain $f \in C^{q}(Q, G)$ is called normalized provided $f\left(x_{1}, \cdots, x_{q}\right)$ $=0$ whenever $x_{i}=1$ for at least one index $i=1, \cdots, q$. The normalized cochains form a subgroup $C_{n}^{q}$ of $C^{q}$. Since the coboundary of a normalized cochain is again normalized, the groups $\left\{C_{n}^{q}\right\}$ define cohomology groups $H_{n}^{q}$ the same way as the sequence $\left\{C^{q}\right\}$ defines $H^{a}$. Since every normalized cocycle also is a cocycle in the ordinary sense, a "natural" homomorphism $H_{n}^{a} \rightarrow H^{q}$ is induced. We have (Eilenberg-MacLane [15])

(2.1) The natural homomorphism $H_{n}^{q}(Q, G) \rightarrow H^{q}(Q, G)$ maps the cohomology group of $Q$ defined using normalized cochains isomorphically onto the cohomology group of $Q$.

The same is true for two-sided operators.

3. The groups $H^{0}, H^{1}$. A 0 -cochain $f \in C^{0}(Q, G)$ is by definition an element $g \in G$. Since $(\delta f)(x)=x g-g$ it follows that $f$ is a cocycle if and only if $x g=g$. Since by definition $B^{0}(Q, G)=0$ we have

(3.1) The 0th cohomology group $H^{0}(Q, G)$ is the subgroup of those elements of $G$ on which $Q$ operates simply.

A 1 -cochain $f \in C^{1}(Q, G)$ is a function $f: Q \rightarrow G$ while its coboundary is

$$
(\delta f)\left(x_{1}, x_{2}\right)=x_{1} f\left(x_{2}\right)-f\left(x_{1} x_{2}\right)+f\left(x_{1}\right),
$$

thence $f$ is a 1-cocycle if and only if

$$
f\left(x_{1} x_{2}\right)=f\left(x_{1}\right)+x_{1} f\left(x_{2}\right) .
$$

Such functions $f$ are known as crossed homomorphisms of $Q$ onto $G$. In order that $f \in B^{1}(Q, G)$ we must have $f(x)=x g-x$ for some constant $g \in G$. Such crossed homomorphisms are called principal homomorphisms. Hence

(3.2) The first cohomology group $H^{1}(Q, G)$ is the group of crossed homomorphisms of $Q$ into $G$ reduced modulo the principal homomorphisms. If the operators of $Q$ on $G$ are simple (that is, if $x g=g$ for all $x$ and $g)$ then $H^{1}(Q, G)$ is the group Hom $(Q, G)$ of all homomorphisms of $Q$ into $G$.

The first cohomology group is useful in connection with the following concepts. Let $G$ and $P$ be groups with the group $Q$ as a group of left operators. It is not assumed that $P$ is abelian and it will be 
written multiplicatively. A pair $(E, \phi)$ will be called a $Q$-enlargement of $P$ by $G$ provided: $\left(1^{\circ}\right) E$ is a group with $Q$ as left operators, $\left(2^{\circ}\right) G$ is a subgroup of $E$ and a direct summand of $E,\left(3^{\circ}\right)$ the operators of $Q$ on $G$ agree with the operators of $Q$ on $E,\left(4^{\circ}\right) \phi$ is an operator homomorphism of $E$ onto $P$ with $G$ as kernel. Two $Q$-enlargements $\left(E_{1}, \phi_{1}\right),\left(E_{2}, \phi_{2}\right)$ of $P$ by $G$ are called equivalent if there is an operator isomorphism $\tau: E_{1} \approx E_{2}$ leaving $G$ pointwise fixed and such that $\phi_{2} \tau=\phi_{1}$.

A $Q$-enlargement $(E, \phi)$ of $P$ by $G$ is said to be inessential if there is an operator homomorphism $\psi: P \rightarrow E$ such that $\phi \psi$ is the identity. The inessential enlargements form an equivalence class.

Multiplication of equivalence classes of enlargements is defined as follows. Given $Q$-enlargements $\left(E_{1}, \phi_{1}\right),\left(E_{2}, \phi_{2}\right)$ of $P$ by $G$, consider the group $E_{1} \times E_{2}$ and its subgroup $F$ consisting of pairs $\left(e_{1}, e_{2}\right)$ with $\phi_{1}\left(e_{1}\right)=\phi_{2}\left(e_{2}\right)$. Clearly $F$ contains the group $G \times G$. Let $\bar{G}$ be the subgroup of $G \times G$ defined by all the pairs of the form $\left(g, g^{-1}\right)$. The group $G \times G / \bar{G}$ can be identified with the group $G$ by identifying each coset $\left(g_{1}, g_{2}\right) \bar{G}$ with the element $g_{1}+g_{2}$. Now let $E=F / \bar{G}$ and define $\phi: E \rightarrow P$ by $\phi\left[\left(e_{1}, e_{2}\right) \bar{G}\right]=\phi_{1}\left(e_{1}\right)=\phi_{2}\left(e_{2}\right)$, also define the operators of $Q$ on $E$ by setting $x\left[\left(e_{1}, e_{2}\right) \bar{G}\right]=\left(x e_{1}, x e_{2}\right) \bar{G}$. With these definitions $(E, \phi)$ is a $Q$-enlargement of $P$ by $G$ which we define as the product $\left(E_{1}, \phi_{1}\right) \otimes\left(E_{2}, \phi_{2}\right)$.

(3.3) The equivalence classes of enlargements of $P$ by $G$ over $Q$ form a commutative group $\operatorname{Enl}(Q, P, G)$. The equivalence class of inessential enlargements is the zero element of this group.

To exhibit the connection of the group Enl $(P, G, Q)$ with cohomology theory we consider homomorphisms $\theta: P \rightarrow G$. With addition and operators defined by

$$
\left(\theta_{1}+\theta_{2}\right)(p)=\theta_{1}(p)+\theta_{2}(p), \quad(x \theta)(p)=x\left[\theta\left(x^{-1} p\right)\right]
$$

the homomorphisms $\theta$ form an abelian group $\operatorname{Hom}(P, G)$ with $Q$ as left operators.

Let $(E, \phi)$ be a $Q$-enlargement of $P$ by $G$. Since $G$ is a direct summand of $E$ and $\phi$ maps $E$ onto $P$ with $G$ as kernel, there exists a homomorphism $\psi: P \rightarrow E$ such that $\phi \psi=$ identity. Unless the enlargement is inessential $\psi$ cannot be chosen to be an operator homomorphism. The expression

$$
x \psi\left(x^{-1} p\right)-\psi(p), \quad x \in Q, p \in P,
$$

measures the deviation of $\psi$ from being an operator homomorphism. An application of $\phi$ shows that $\left(^{*}\right)$ is in $G$, and for a fixed $x \in Q,\left(^{*}\right)$ gives a homomorphism $\psi^{*}(x)$ of $P$ into $G$. Thus $\psi^{*}(x) \in \operatorname{Hom}(P, G)$ and $\psi^{*} \in C^{1}(Q$, Hom $(P, G))$. A computation yields 


$$
\begin{aligned}
\psi^{*}(x y)(p) & =x y \psi\left(y^{-1} x^{-1} p\right)-\psi(p) \\
& =x\left[y \psi\left(y^{-1} x^{-1} p\right)-\psi\left(x^{-1} p\right)\right]+\left[x \psi\left(x^{-1} p\right)-\psi(p)\right] \\
& =x\left[\psi^{*}(y)\left(x^{-1} p\right)\right]+\psi^{*}(x)(p) .
\end{aligned}
$$

Thus

$$
\psi^{*}(x y)=x \psi^{*}(y)+\psi^{*}(x),
$$

that is, $\delta \psi^{*}=0$ and $\psi^{*} \in Z^{1}(Q$, Hom $(P, G))$. If $\psi$ is replaced by another homomorphism $\psi_{1}: P \rightarrow E$ such that $\phi \psi_{1}=$ identity then $\psi_{1}-\psi=\theta$ $\in \operatorname{Hom}(P, G)=C^{0}(Q$, Hom $(P, G))$, and a computation shows that $\psi_{1}^{*}-\psi^{*}=\delta \theta$. Thus the cohomology class $\left\{\psi^{*}\right\}$ of the cocycle $\psi^{*}$ is independent of the choice of $\psi$ and is completely determined by the enlargement $(E, \phi)$. This yields a map Enl $(Q, P, G)$ $\rightarrow H^{1}(Q$, Hom $(P, G))$. It is easy to see that this correspondence yields an isomorphism onto.

(3.4) The group $\operatorname{Enl}(Q, P, G)$ of equivalence classes of $Q$-enlargements of $P$ by $G$ is isomorphic with the cohomology group $H^{1}(Q, \operatorname{Hom}(P, G))$.

The previous argument becomes more transparent if instead of defining Hom $(P, G)$ as a group with $Q$ as left operators, we define Hom $(P, G)$ with $Q$ as two-sided operators as follows

$$
(x \theta)(p)=x \theta(p), \quad(\theta x)(p)=\theta(x p) .
$$

The "deviation" $\psi^{*}(x)$ is then defined as

$$
\psi^{*}(x)(p)=x \psi(p)-\psi(x p) .
$$

Then

$$
\psi^{*}(x y)=x \psi^{*}(y)+\phi^{*}(x) y
$$

and $\psi^{*}$ is a cocycle following the formula with two-sided operators. The discussion with left-operators corresponds then to the discussion with two-sided operators by means of the mapping $\phi$ of $\$ 2$.

This discussion concerning enlargements is capable of the following generalization. Suppose that in addition to the group $Q$ another group $R$ is given that also operates on $G$ and $P$ on the right. We shall assume in addition that the operators of $Q$ and $R$ commute, that is, that

$$
(x g) r=x(g r), \quad(x p) r=x(p r)
$$

for $x \in Q, r \in R, g \in G, p \in P$. We may then consider pairs $(E, \phi)$ which are simultaneously $Q$ and $R$-enlargements and such that the operators of $Q$ and $R$ on $E$ also commute. We shall then say that $(E, \phi)$ is a $Q$ - $R$-enlargement. The equivalence classes of $Q-R$ - 
enlargements which are inessential as $R$-enlargements form a commutative group $\operatorname{Enl}_{R}(Q, P, G)$. The zero element of this group consists of the equivalence class of $Q-R$-enlargements $(E, \phi)$ which are $Q-R$-inessential, that is, of those for which there exists a homomorphism $\psi: P \rightarrow E$ which is both a $Q$ - and $R$-operator homomorphism such that $\phi \psi=$ identity. Replacing in the previous discussion all the homomorphisms $\theta: P \rightarrow G$ by $R$-operator homomorphisms, we find that the group $\operatorname{Enl}_{R}(Q, P, G)$ is isomorphic with the cohomology group $H^{1}\left(Q, \operatorname{Hom}_{R}(P, G)\right)$ where $\operatorname{Hom}_{R}(P, G)$ is the group of $R$ operator homomorphisms of $P$ into $G$ with the operators of $Q$ defined as before.

The above discussion of enlargements is new, but is a close analogue of a similar discussion for associative algebras (Hochschild [24]).

4. Group extensions. The discussion of the 2 nd and 3rd cohomology groups is closedly connected with the problem of group extensions (Eilenberg-MacLane [16]).

Consider a group $E$, an invariant subgroup $K$ of $E$ and a homomorphism $\phi: E \rightarrow Q$ mapping $E$ onto $Q$ with kernel $K$. Let $G$ denote the center of the group $K$. For every $e \in E$ the mapping

$$
k \rightarrow e k e^{-1}
$$

is an automorphism of $K$; thus a homomorphism $E \rightarrow A(K)$ of $E$ into the group $A(K)$ of automorphisms of $K$ is defined. The subgroup $K$ maps into the subgroup $I(K)$ of inner automorphisms of $K$. There results a homomorphism

$$
\theta: Q \rightarrow A(K) / I(K) .
$$

Replacing $k \in K$ by $g \in G$ we also find a homomorphism $Q \rightarrow A(G)$. This means that $Q$ operates on $G$. From now on we shall assume that $Q, G$ and the operators of $Q$ on $G$ are fixed. The pair $(E, \phi)$ is then called an extension of $Q$ belonging to the center $G$, the pair $(K, \theta)$ is then called the kernel of the extension $(E, \phi)$.

Two extensions $\left(E_{1}, \phi_{1}\right)$ and $\left(E_{2}, \phi_{2}\right)$ are called equivalent if there is an isomorphism $\tau$ of $E_{1}$ onto $E_{2}$ such that $\phi_{2} \tau=\phi_{1}$ and $\tau(g)=g$ for each $g \in G$.

Multiplication of equivalence classes of extensions is defined as follows. Given extensions $\left(E_{1}, \phi_{1}\right),\left(E_{2}, \phi_{2}\right)$ of $Q$ belonging to the center $G$, consider the group $E_{1} \times E_{2}$ and its subgroup $F$ consisting of pairs $\left(e_{1}, e_{2}\right)$ with $\phi_{1}\left(e_{1}\right)=\phi_{2}\left(e_{2}\right)$. Clearly $F$ contains the group $G \times G$. Let $\bar{G}$ be the subgroup of $G \times G$ defined by all pairs of the form $\left(g, g^{-1}\right)$. The group $G \times G / \bar{G}$ can be identified with the group $G$ by identifying each coset $\left(g_{1}, g_{2}\right) \bar{G}$ with the element $g_{1}+g_{2}$. Now let $E=F / \bar{G}$ and 
define $\phi: E \rightarrow Q$ by $\phi\left[\left(e_{1}, e_{2}\right) \bar{G}\right]=\phi_{1}\left(e_{1}\right)=\phi_{2}\left(e_{2}\right)$. The extension $(E, \phi)$ is defined to be the product $\left(E_{1}, \phi_{1}\right) \otimes\left(E_{2}, \phi_{2}\right)$.

(4.1) The equivalence classes of extensions of $Q$ belonging to the center $G$ (with prescribed operators of $Q$ on $G$ ) form a commutative and associative system $\mathcal{E}(Q, G)$.

Extensions $(E, \phi)$ in which $K=G$ (that is, the kernel is abelian) form a multiplicative subsystem $\mathcal{A}(Q, G)$ of $\mathcal{E}(Q, G)$. The extension $(E, \phi)$ with $K=G$ is said to split provided there exists a homomorphism $\psi: Q \rightarrow E$ such $\phi \psi=$ identity. The splitting extensions constitute an equivalence class.

(4.2) The extensions with $K=G$ form an abelian subgroup $\mathcal{A}(Q, G)$ of $\mathcal{E}(Q, G)$. The zero element of $\mathcal{A}(Q, G)$ is also a zero element for $\mathcal{E}(Q, G)$ and is the equivalence class of the splitting extensions.

The extensions $(E, \phi)$ with $K=G$ are called the group extensions of $Q$ by $G$, and $\mathcal{A}(Q, G)$ is called the group of group extensions of $Q$ by $G$ (with the prescribed operators of $Q$ on $G$ ). The first result connecting group extension and cohomology is the following.

(4.3) The group $\mathcal{A}(Q, G)$ of extensions of $Q$ by $G$ is isomorphic with the second cohomology group $H^{2}(Q, G)$.

The nature of this isomorphism will be explained in detail since it is typical of the method by which the cohomology theory enters into a problem of algebra.

Let $(E, \phi)$ be an element of an equivalence class of $\mathcal{A}(Q, G)$. Then $\phi: E \rightarrow Q$ maps $E$ onto $Q$ with $G$ as kernel and with prescribed operators. In an effort to split the extension we consider an arbitrary map $u: Q \rightarrow E$ such that $\phi u=$ identity. If the map $u$ is a homomorphism the extension splits. In general $u$ will not be a homomorphism and the deviation of $u$ from a homomorphism is a function $f(x, y)$ defined by

$$
u(x) u(y)=f(x, y) u(x y)
$$

for $x, y \in Q$. Clearly $f(x, y) \in G$ so that $f \in C^{2}(Q, G)$. A computation yields

$$
\begin{aligned}
& {[u(x) u(y)] u(z)=f(x, y) f(x y, z) u(x y z),} \\
& u(x)[u(y) u(z)]=[x f(y, z)] f(x, y z) u(x y z) .
\end{aligned}
$$

Hence the associativity of $E$ implies

$$
f(x, y) f(x y, z)=[x f(y, z)] f(x, y z)
$$

and since all the elements are in $G$ we can pass to additive notation:

that is,

$$
x f(y, z)-f(x y, z)+f(x, y z)-f(x, y)=0
$$




$$
\delta f=0
$$

and $f \in Z^{2}(Q, G)$. Thus the deviation $f$ (called factor set) is a cocycle; the cohomology class $\{f\}$ of this cocycle is independent of the choice of the representative function $u$ and also of the choice of the extension $(E, \phi)$ within its equivalence class. This yields a map $A(Q, G)$ $\rightarrow H^{2}(Q, G)$ which is easily verified to be an isomorphism onto. The cohomology class $\{f\}=F^{2}(E, \phi)$ attached to the extension $(E, \phi)$ may be regarded as the obstruction against splitting $(E, \phi)$; the extension splits if and only if $F^{2}(E, \phi)=0$.

The study of extensions of $Q$ by $G$ also leads to an interpretation of the first cohomology group $H^{1}(Q, G)$ (Baer [2]). Let $(E, \phi)$ be an extension of $Q$ by $G$. In the group $A(E)$ of automorphisms of $E$ consider the subgroup $A_{1}$ consisting of automorphisms $\alpha$ which satisfy $\phi \alpha=\phi$. Then setting $m(e)=\alpha(e) e^{-1}$ yields a cocycle $m \in Z^{1}(E, G)$ with $E$ operating on $G$ as inner automorphisms. In $A_{1}$ consider the subgroup $A_{2}$ of all automorphisms which leave $G$ pointwise fixed. If $\alpha \in A_{2}$ then there is a unique cocycle $n \in Z^{1}(Q, G)$ such that $n \phi=m$. It is easy to see that $A_{2}$ is abelian and that the correspondence $\alpha \rightarrow n$ yields an isomorphism $A_{2} \approx Z^{1}(Q, G)$. Under this isomorphism $B^{1}(Q, G)$ corresponds to the subgroup $A_{3}$ of $A_{2}$ consisting of all automorphisms $e \rightarrow g e g^{-1}, g \in G$. Thus $A_{2} / A_{3} \approx H^{1}(Q, G)$.

Before we proceed with the discussion of extensions whose kernel is not abelian, we must devote some attention to kernels.

5. Kernels. A $Q$-kernel is a pair $(K, \theta)$ where $K$ is a group and

$$
\theta: Q \rightarrow A(K) / I(K)
$$

is a homomorphism. If $G$ is the center of $K$, then $\theta$ induces a homomorphism

$$
Q \rightarrow A(G),
$$

that is, $Q$ operates on $G$. We shall only consider $Q$-kernels with a fixed center $G$ and fixed operators of $Q$ on $G$. Equivalence of kernels is defined in the obvious way. Multiplication of equivalence classes of $Q$-kernels is defined as follows. Given kernels $\left(K_{1}, \theta_{1}\right),\left(K_{2}, \theta_{2}\right)$ consider the group $K=K_{1} \times K_{2} / \bar{G}$ where $G$ is the subgroup of $G \times G$ considered earlier. The group $G \times G / \bar{G}$, that we have identified earlier with $G$, is then the center of $K$. Let $x \in Q$ and let $\theta_{i}(x)=\alpha_{i} I\left(K_{i}\right)$ where $\alpha_{i} \in A\left(K_{i}\right), i=1,2$. Since $\alpha_{1}$ and $\alpha_{2}$ coincide on $G$ it follows that $\left(\alpha_{1}, \alpha_{2}\right)$ is an automorphism of $K_{1} \times K_{2}$ which leaves $\bar{G}$ invariant. Thus $\left(\alpha_{1}, \alpha_{2}\right)$ defines an automorphism $\alpha$ of $K$. We define $\theta(x)=\alpha I(K)$. The resulting kernel $(K, \theta)$ is the product $\left(K_{1}, \theta_{1}\right) \otimes\left(K_{2}, \theta_{2}\right)$. 
(5.1) The multiplication of equivalence classes of Q-kernels is commutative and associative. The kernel $(K, \theta)$ with $K=G$ acts as a unit.

We have observed in $\$ 4$ that each extension $(E, \phi)$ of $Q$ belonging to the center $G$ determines a $Q$-kernel $(K, \theta)$. However not every kernel is the kernel of an extension. This defines a subclass of extendible kernels, that is, of those $Q$-kernels which are kernels of extensions. Two $Q$-kernels $\left(K_{1}, \theta_{1}\right)$ and $\left(K_{2}, \theta_{2}\right)$ are called similar provided there exist extendible $Q$-kernels $\left(L_{1}, \gamma_{1}\right),\left(L_{2}, \gamma_{2}\right)$ such that $\left(K_{1}, \theta_{1}\right) \otimes\left(L_{1}, \gamma_{1}\right)$ is equivalent with $\left(K_{2}, \theta_{2}\right) \otimes\left(L_{2}, \gamma_{2}\right)$. Since the product of extendible kernels is extendible, similarity classes of kernels can be multiplied.

(5.2) The similarity classes of Q-kernels with center $G$ (and prescribed operators of $Q$ on $G$ ) form an abelian group $K(Q, G)$. The zero element of this group consists of the class of extendible $Q$-kernels.

The main result concerning kernels is the following (Eilenberg-MacLane [16]).

(5.3) The group $K(Q, G)$ is isomorphic with the 3rd cohomology group $H^{3}(Q, G)$.

This isomorphism is established by a method similar to that used for (4.3). Let $(K, \theta)$ be a $Q$-kernel. For each $x \in Q$ select $\alpha(x) \in A(Q)$ such that $\theta(x)=\alpha(x) I(K)$. Then

$$
\alpha(x) \alpha(y)=\beta(x, y) \alpha(x y)
$$

where $\beta(x y) \in I(K)$. Select $h(x, y) \in K$ so that

$$
h(x, y) k h(x, y)^{-1}=\beta(x y)(k) .
$$

Finally define $f(x, y, z)$ by

$$
[\alpha(x), h(y, z)] h(x, y z)=f(x, y, z) h(x, y) h(x y, z) .
$$

In the noncommutative sense $f$ is then the coboundary of $h . A$ computation shows that $f$ is in the center of $K$, that is, in $G$, and that $f$ is a cocycle $f \in Z^{3}(Q, G)$. The characteristic cohomology class $\{f\}$ $=F^{3}(K, \theta)$ is independent of the choices made in the definition of $f$, and of the choice of $(K, \theta)$ within its equivalence class.

(5.4) $A$ Q-kernel $(K, \theta)$ with center $G$ is extendible if and only if its characteristic cohomology class $F^{3}(K, \theta) \in H^{3}(Q, G)$ is zero.

Since the characteristic cohomology classes add when kernels are multiplied it follows that $(K, \theta) \rightarrow F^{3}(K, 0)$ is a homomorphism $K(Q, G) \rightarrow H^{3}(Q, G)$. Proposition (5.3) is proved by showing that this map is an isomorphism onto.

A remark should be made concerning the normalization of the cocycles considered in $\$ 4$ and this section. The cocycle of $\$ 4$ will 
be normalized if and only if we select the representative function $u$ so that $u(1)=1$. In order to insure that the 3-dimensional cocycle of a kernel be normalized we select $\alpha(1)=1$ so that $\beta(1, y)=\beta(x, 1)=1$. Then select $h$ so that $h(1, y)=h(x, 1)=1$.

For further results concerning kernels see MacLane [32].

6. Extensions with a non-abelian kernel. Let $(K, \theta)$ be a $Q$-kernel with center $G$. Assume further that $F^{3}(K, \theta)=0$ so that there is at least one extension $\left(E_{0}, \phi_{0}\right)$ of $Q$ with $(K, \theta)$ as kernel. Let $\left[E_{0}, \phi_{0}\right]$ be the element of $\mathcal{E}(Q, G)$ determined by $\left(E_{0}, \phi_{0}\right)$. The main results then are (Eilenberg-MacLane [16]):

(6.1) The map $(E, \phi) \rightarrow\left(E_{0}, \phi_{0}\right) \otimes(E, \phi)$, where $(E, \phi)$ is any extension of $Q$ with $G$ as kernel, establishes a 1-1 map of the group $\mathcal{A}(Q, G)$ onto the totality of equivalence classes of extensions of $Q$ with kernel equivalent with $(K, \phi)$. The latter form then a coset $\left[E_{0}, \phi_{0}\right] \otimes \mathcal{A}(Q, G)$.

Combining (6.1) with (4.3) yields:

(6.2) If the $Q$-kernel $(K, \theta)$ is extendible then the equivalence classes of extensions $(E, \phi)$ of $Q$ with kernel $(K, \theta)$ are in a 1-1 correspondence with the elements of the group $H^{2}(Q, G)$ where $G$ is the center of $K$ and the operators of $Q$ on $G$ are induced by $\theta$.

7. Non-associative systems. The fact that the deviation $f$ defined in $\S 4$ by the equation $u(x) u(y)=f(x, y) u(x y)$ is a 2-cocycle is equivalent with the associativity law in the group $E$. This suggests that higher-dimensional cocycles may be obtained as deviations from the associativity law in suitable multiplicative systems. This point of view has been explored (Eilenberg-MacLane [17]) and leads to theorems generalizing (4.3) and employing the higher cohomology groups.

Let $L$ be a loop (that is, a non-associative group). Given $a, b, c \in L$ define the associator $A(a, b, c) \in L$ by the equation

$$
a(b c)=A(a, b, c)[(a b) c] .
$$

The associator is then a measure of the deviation from associativity. To illustrate the tendency of the associator to behave like a 3-cocycle assume that $L$ is commutative and that the associativity law holds whenever one of the three elements is itself an associator. Then

$$
\begin{aligned}
a[b(c d)] & =A(a, b, c d)(a b)(c d)=A(a, b, c d) A(a b, c, d)[(a b) c] d, \\
a[b(c d)] & =A(b, c, d) a[(b c) d]=A(b, c, d) A(a, b c, d)[a(b c)] d \\
& =A(b, c, d) A(a, b c, d) A(a, b, c)[(a b) c] d .
\end{aligned}
$$

Thus (using additive notation) 


$$
A(b, c, d)-A(a b, c, d)+A(a, b c, d)-A(a, b, c d)+A(a, b, c)=0
$$

which shows that $A$ is a suitable generalization of a 3-cocycle.

Higher associators are defined inductively as

$$
A\left(a_{1}, \cdots, a_{2 n+1}\right)=A\left(a_{1}, \cdots, a_{2 n-2}, A\left(a_{2 n-1}, a_{2 n}, a_{2 n+1}\right)\right)
$$

and also have a tendency to behave like cocycles.

The generalization of (4.3) is achieved by the study of prolongations. A prolongation of $Q$ by $G$ (with given operators of $Q$ on $G$ ) is a pair $(L, \phi)$ satisfying the following five conditions:

(i) $L$ is a loop containing $G$ as a subgroup, G,

(ii) $\phi$ is a homomorphism of $L$ onto $Q$ with a kernel $K$ containing

(iii) $A(k, a, b)=1=A(a, k, b)$ for $a, b \in L, k \in K$,

(iv) $k A=A k$ for any $k \in K$ and any associator $A=A(a, b, c)$,

(v) $a g=(\phi(a) g) a$ for $a \in L, g \in G$.

The equivalence and multiplication of prolongations is defined as in \$4. The equivalence classes of prolongations of $Q$ by $G$ form then a commutative and associative system $P(Q, G)$. The splitting extension of $Q$ by $G$ acts as a unit for $P(Q, G)$.

The non-associativity of $L$ in a prolongation $(L, \phi)$ is further controlled by the consideration of the following four classes defined by the following conditions

$O_{n}^{L}:$

$$
\begin{aligned}
& A\left(a_{1}, \cdots, a_{2 n}, a\right)=1, \\
& A\left(a_{1}, \cdots, a_{2 n}, k\right)=1, \\
& A\left(a_{1}, \cdots, a_{2 n}, a\right)=G, \\
& A\left(a_{1}, \cdots, a_{2 n}, k\right)=G,
\end{aligned}
$$

where $n>0, a_{1}, \cdots, a_{2 n}, a \in L$ and $k \in K$. In addition we define the class $R_{n}$ consisting of those prolongations $(L, \phi)$ of $Q$ by $G$ which contain a prolongation $\left(L^{\prime}, \phi^{\prime}\right)$ of $Q$ by $G$ (that is, $L^{\prime} C L$ and $\phi^{\prime}$ agrees with $\phi$ on $L^{\prime}$ ) of class $O_{n}^{K}$. We also define the class $G_{0}^{K}$ by the condition $K=G$.

Next we consider the class $O_{n}^{K} \cap G_{n}^{L}$. This class contains both $O_{n}^{L}$ and $G_{n-1}^{K}$ and therefore contains the class $Q_{n}^{K} \otimes G_{n-1}^{K}$ of products of prolongation in $O_{n}^{L}$ and $G_{n-1}^{K}$. We then define as in $\$ 5$ the similarity classes of $O_{n}^{K} \cap G_{n}^{L}$ modulo $Q_{n}^{L} \otimes G_{n-1}^{K}$.

(7.1) The similarity classes of $O_{n}^{K} \cap G_{n}^{L}$ modulo $O_{n}^{L} \otimes G_{n-1}^{K}$ form a group $O_{n}^{K} \cap G_{n}^{L} / O_{n}^{L} \otimes G_{n-1}^{K}$ which is isomorphic with the cohomology group $H^{2 n-1}(Q, G), n>0$.

Similarly 
(7.2) The similarity classes of $O_{n-1}^{L} \cap G_{n}^{K}$ modulo $R_{n} \otimes G_{n}^{L}$ form a group $O_{n-1}^{L} \cap G_{n}^{K} / R_{n} \otimes G_{n}^{L}$ isomorphic with the cohomology group $H^{2 n+2}(Q, G)$, $n>0$.

In the case $n=0$ the various classes can be interpreted so that this result reduces to the group extension case (4.3).

Let us consider the case $n=1$ of (7.1) in more detail. A prolongation $(L, \phi)$ is in the class $O_{1}^{K} \cap G_{1}^{L}$ if $A(a, b, k)=1$ and if $A(a, b, c) \in G$. Thus we obtain the class $O_{1}^{K} \cap G_{1}^{L}$ by replacing conditions (iii) and (iv) by

(iii)' $A(k, a, b)=A(a, k, b)=A(a, b, k)=1$ for $a, b \in L, k \in K$,

(iv)' all associators of $L$ are in $G$.

The class $O_{1}^{L}$ consists of those prolongations $(L, \phi)$ for which $L$ is associative, that is, a group, while $G_{0}^{K}$ is defined by the condition $K=G$. The similarity classes of $O_{1}^{K} \cap G_{1}^{L}$ modulo $O_{1}^{L} \otimes G_{0}^{K}$ form then a group isomorphic with $H^{3}(Q, G)$. This characterization of $H^{3}(Q, G)$ is quite different from the characterization of $H^{3}(Q, G)$ as the group of $Q$-kernels $K(Q, G)$ asserted in (5.3). It is very likely that some sort of nonassociative generalization of the concept of a kernel and of the group $K(Q, G)$ exists, that will give a characterization of the cohomology groups $H^{q}(Q, G)$ for $q>3$.

8. Connections with Galois theory. Let $P$ be a field, $N$ a finite, separable, and normal extension of $P$, and $Q$ the Galois group of $N$ over $P$. Each element $\lambda \in Q$ is then an automorphism $\lambda: N \rightarrow N$ leaving $P$ pointwise fixed. Thus $Q$ acts as a group of operators on both the additive group $N^{+}$of $N$ and the multiplicative group $N^{\times}$of the elements of $N$ different from zero. Thus we are in a position to study the cohomology groups $H^{q}\left(Q, N^{+}\right)$and $H^{q}\left(Q, N^{\times}\right)$. It is easy to verify that $H^{q}\left(Q, N^{+}\right)=0$ for all dimensions $q$. Thus our attention will be centered on the group $H^{q}\left(Q, N^{\times}\right)$, from now on written as $H^{q}(Q, N)$. The base field $P$ is not indicated in this notation as it simply is the field of all fixed points of $N$ under the automorphisms of the group $Q$.

The "principal genus theorem in the small" asserts that

$$
H^{1}(Q, N)=0 \text {. }
$$

The cohomology groups $H^{2}(Q, N)$ and $H^{3}(Q, N)$ enter the picture in connection with the theory of simple algebras and crossed products.

Consider the totality of all central simple algebras $A$ over $P$ with the Kronecker product over $P$ as multiplication. The similarity classes modulo algebras which are full matrix algebras over $P$ form a group $A(P)$ called the Brauer group of algebra classes over $P$. 
Let $N$ be a finite separable and normal extension of $P$. For each algebra $A$ over $P$ construct the scalar extension $A_{N}=A \times{ }_{P} N$ which is an algebra over $N$. If $A$ is central simple over $P$ then $A_{N}$ is central simple over $N$. The correspondence $A \rightarrow A_{N}$ yields a homomorphism

$$
A(P) \rightarrow \mathcal{A}(N) \text {. }
$$

The kernel of this homomorphism will be denoted by $\mathscr{A}^{N}(P)$ and consists of the algebra classes over $P$ for which $N$ is a splitting field.

(8.2) The second cohomology group $H^{2}(Q, N)$ is isomorphic with the group $A^{N}(P)$ of algebra classes over $P$ split by $N$.

This is a reformulation of a classical result and is obtained by constructing for each $f \in Z^{2}(Q, N)$ a crossed product algebra $A=(f, Q, N)$ as follows. $A$ is a vector space over $N$ generated by symbols $u(x)$ corresponding to elements $x \in Q$. The multiplication table is given by

$$
[a u(x)][b u(y)]=a(x b) f(x, y) u(x, y),
$$

or equivalently

$$
u(x) b=(x b) u(x), \quad u(x) u(y)=f(x, y) u(x y) .
$$

With $f$ assumed normalized, $u(1)$ is the unit element of $A$. The algebra $A$ is central simple over $P$ and has $N$ as splitting field. Further every central simple algebra over $P$ which is split by $N$ is similar to a crossed product $(f, Q, N)$ with $f$ determined uniquely modulo coboundaries. This leads to (8.2) (see for instance Artin-NesbittThrall [1]).

The image of the homomorphism (*) will be denoted by $A_{N}(P)$ and will be used presently.

A central simple algebra $A$ over $N$ will be called $Q$-normal provided every automorphism in the group $Q$ can be extended to an automorphism of the algebra $A$. Teichmüller [33] has studied such algebras and has shown that if $A$ is $Q$-normal then any algebra similar to $A$ is $Q$-normal. Thus the $Q$-normal algebras determine a subgroup $\mathcal{N}_{Q}(N)$ of $A(N)$. It is easy to see that

$$
A_{N}(P) \subset \mathcal{N}_{Q}(N) \text {. }
$$

The connection between $Q$-normal algebras and the cohomology group $H^{3}(Q, N)$ was introduced by Teichmüller who has defined a homomorphism

$$
T^{3}: \mathcal{N}_{Q}(N) \rightarrow H^{3}(Q, N) .
$$

This correspondence could be described in the language of $\$ 5$ as follows. 
Let $A$ be $Q$-normal and let $K$ be the group of regular elements of $A$. Then $N^{\times}$is the center of $K$. Since each 'automorphism $x \in Q$ can be extended to an automorphism of $A$ each $x \in Q$ gives rise to subset $\theta(x)$ of the automorphism group $A(K)$. Since $A$ is central simple over $N$, any two extensions of $x$ differ by an inner automorphism and $0(x)$ is an element of $A(K) / I(K)$. Thus $\theta: Q \rightarrow A(K) / I(K)$ and the pair $(K, \theta)$ is a $Q$-kernel with $N^{\times}$as center. The cohomology class $T^{3}(A)$ is then the characteristic cohomology class of the kernel $(K, \theta)$

$$
T^{3}(A)=F^{3}(K, \theta) \in H^{3}(Q, N) .
$$

Teichmüller [33] has proved that $\mathcal{A}_{N}(P)$ is the kernel of the homomorphism $T^{3}$, and thus that the group $\mathcal{N}_{Q}(N) / \mathcal{A}_{N}(P)$ is isomorphic with a subgroup of $H^{3}(Q, N)$. This subgroup was subsequently determined by Eilenberg-MacLane [18] as follows.

Let $K$ be a finite normal and separable extension of $P$ such that $P \subset N \subset K$ and let $G$ be the Galois group of $K$ over $P$. Each $\alpha \in G$ defines then an element $\phi(\alpha) \in Q$. Given a cochain $f \in C^{q}(Q, N)$ define the "lifted" cochain $\Lambda_{K} f \in C^{q}(G, K)$ by setting

$$
\Lambda_{K} f\left(\alpha_{1}, \cdots, \alpha_{q}\right)=f\left(\phi\left(\alpha_{1}\right), \cdots, \phi\left(\alpha_{q}\right)\right) .
$$

This lifting operation defines a homomorphism

$$
\Lambda_{K}: H^{q}(Q, N) \rightarrow H^{q}(G, K) .
$$

A cohomology class in the kernel of $\Lambda_{K}$ is said to be split by $K$ or to have ceiling $K$. The cohomology classes in $H^{q}(Q, N)$ which have a ceiling form a subgroup $H_{0}^{q}(Q, N)$. For $q=2$ this group can be shown to be trivial.

(8.3) The homomorphism $T^{3}$ maps the group of Q-normal algebra classes over $P$ onto the subgroup $H_{0}^{q}(Q, N)$ of $H^{q}(Q, N)$. The kernel of $T^{3}$ is the group $\mathcal{A}_{N}(P)$. Thus

$$
\mathcal{N}_{Q}(N) / \mathcal{A}_{N}(P) \approx H_{0}^{3}(Q, N) .
$$

The proof of (8.3) requires a detailed analysis of the $Q$-normality of crossed products. The computations are considerable and the normalization conditions discussed in $\$ 2$ are used extensively. See also MacLane [31] for an alternative proof of a main lemma.

In the case when $P$ is an algebraic number field, MacLane [30] has proved (without appeal to cohomology theory) that the group $\mathcal{N}_{Q}(N) / \mathcal{A}_{N}(P)$ is cyclic of order $s$ where $s$ is determined by the arithmetric properties of the extension $N$ of $P$. Examples can be found with $s>1$; therefore $H_{0}^{3}(Q, N) \neq 0$ in this case.

9. The first reduction theorem. We now turn to the intrinsic prop- 
erties of the cohomology groups of groups. The main problem consists in devising a method for computing the cohomology groups. In this connection the so-called "reduction theorems" are of great assistance. We call a "reduction theorem" any theorem that asserts that the cohomology group $H^{q}(Q, G)$ is isomorphic with a lower dimensional cohomology group of $Q$ with a suitably altered coefficient group $G$.

In the reduction theorem that we are about to state it is convenient to use two-sided operators and normalized cochains. We first define $Q$ as a group of two-sided operators on the group of cochains $C^{n}(Q, G)$ as follows

$$
\begin{aligned}
& (x f)\left(x_{1}, \cdots, x_{n}\right)=x\left[f\left(x_{1}, \cdots, x_{n}\right)\right], \\
& (f x)\left(x_{1}, \cdots, x_{n}\right)=x\left[f\left(x_{1}, \cdots, x_{n}\right)\right]-(\delta f)\left(x, x_{1}, \cdots, x_{n}\right) .
\end{aligned}
$$

Next we establish an isomorphism

$$
\tau_{q, n}: C^{q}\left(Q, C^{n}(Q, G)\right) \approx C^{q+n}(Q, G)
$$

by setting

$$
(\tau f)\left(x_{1}, \cdots, x_{q}, y_{1}, \cdots, y_{n}\right)=\left[f\left(x_{1}, \cdots, x_{q}\right)\right]\left(y_{1}, \cdots, y_{n}\right)
$$

for $f \in C^{q}\left(Q, C^{n}(Q, G)\right)$. A computation shows that $\tau_{q, n}$ is an operator isomorphism and that it commutes with the coboundary operator

$$
\delta \tau_{q, n}=\tau_{q-1, n} \delta .
$$

Consequently we obtain isomorphisms

$$
\tau_{q, n}: H^{q}\left(Q, C^{n}(Q, G)\right) \approx H^{q+n}(Q, G),
$$

This is the first reduction theorem (Eilenberg-MacLane [15]); it was originally discovered by Hochschild [22] for cohomology groups of associative algebras.

If $Q$ operates on $G$ only on the left (that is, the right operators of $Q$ on $G$ are simple) we may combine the map $\tau_{g, n}$ with the map $\phi$ of $\$ 2$ and express the reduction theorem in terms of left operators only. Explicitly the formulae take the following form:

$$
\begin{array}{r}
(x f)\left(x_{1}, \cdots, x_{n}\right)=f\left(x_{1}, \cdots, x_{n}\right)-x(\delta f)\left(x^{-1}, x_{1}, \cdots, x_{n}\right), \\
\left(\bar{\tau}_{q, n} f\right)\left(x_{1}, \cdots, x_{q}, y_{1}, \cdots, y_{n}\right)=x_{1} \cdots x_{q}\left[f\left(x_{1}, \cdots, x_{q}\right)\left(y_{1}, \cdots, y_{n}\right)\right] \\
-\delta\left[f\left(x_{1}, \cdots, x_{q}\right)\right]\left(x_{1}, \cdots x_{q}, y_{1}, \cdots, y_{n}\right) .
\end{array}
$$

For $n=1$ the above formulae give

$$
(x f)(y)=x f\left(x^{-1} y\right)-x f\left(x^{-1}\right)
$$




$$
\begin{aligned}
\left(\bar{\tau}_{q} f\right)\left(x_{1}, \cdots, x_{q}, y\right)= & f\left(x_{1}, \cdots, x_{q}\right)\left(x_{1}, \cdots, x_{q} y\right) \\
& -f\left(x_{1} \cdots, x_{q}\right)\left(x_{1} \cdots x_{q}\right)
\end{aligned}
$$

and yield an isomorphism

$$
\bar{\tau}_{q}: H^{q}\left(Q, C^{1}(Q, G)\right) \approx H^{q-1}(Q, G)
$$

with left operators only.

As an application of the reduction theorem we prove:

(9.3) If $Q$ is a free group then $H^{q}(Q, G)=0$ for $q \geqq 2$.

In view of the reduction theorem it suffices to prove that $H^{2}(Q, G)$ $=0$. This amounts to proving that every extension $(E, \phi)$ of $Q$ by $G$ splits. Let $\left\{x_{\alpha}\right\}$ be a base for $Q$; select $\psi\left(x_{\alpha}\right) \in E$ so that $\phi \psi\left(x_{\alpha}\right)=x_{\alpha}$. The map $\psi$ can be extended to a homomorphism $\psi: Q \rightarrow E$. Since $\phi \psi=$ identity, the extension splits.

10. The cup product reduction theorem. Let $G_{1}, G_{2}, G$ be additive abelian groups. We shall say that $G_{1}$ and $G_{2}$ are paired to $G$, if for each $g_{1} \in G_{1}$ and $g_{2} \in G_{2}$ an element $g_{1} \cup g_{2} \in G$ is given such that

$$
\begin{aligned}
& \left(g_{1}+g_{1}^{\prime}\right) \cup g_{2}=g_{1} \cup g_{2}+g_{1}^{\prime} \cup g_{2}, \\
& g_{1} \cup\left(g_{2}+g_{2}^{\prime}\right)=g_{1} \cup g_{2}+g_{1} \cup g_{2}^{\prime} .
\end{aligned}
$$

If in addition $Q$ acts as a group of left operators on $G_{1}, G_{2}, G$ then we also require that for all $x \in Q$

$$
x\left(g_{1} \cup g_{2}\right)=x g_{1} \cup x g_{2} .
$$

Given cochains $f_{1} \in C^{p}\left(Q, G_{1}\right), f_{2} \in C^{q}\left(Q, G_{2}\right)$ we define a cochain $f_{1} \cup f_{2} \in C^{p+q}(Q, G)$ as follows:

$$
\begin{aligned}
\left(f_{1} \cup f_{2}\right)\left(x_{1}, \cdots,\right. & \left.x_{p+q}\right) \\
& =f_{1}\left(x_{1}, \cdots, x_{p}\right) \cup x_{1} \cdots x_{p} f_{2}\left(x_{p+1}, \cdots, x_{p+q}\right) .
\end{aligned}
$$

This yields a pairing of the groups $C_{p}\left(Q, G_{1}\right)$ and $C^{q}\left(Q, G_{2}\right)$ to the group $C^{p+q}(Q, G)$ with the following property

$$
\delta\left(f_{1} \cup f_{2}\right)=\left(\delta f_{1}\right) \cup f_{2}+(-1)^{p} f_{1} \cup \delta f_{2} \text {. }
$$

This yields

$$
\begin{aligned}
\text { cocycle } \cup \text { cocycle } & =\text { cocycle } \\
\text { cocycle } \cup \text { cobound } . & =\text { cobound. } \\
\text { cobound. } \cup \text { cocycle } & =\text { cobound. }
\end{aligned}
$$

and thus leads to a pairing of the cohomology groups $H^{p}\left(Q, G_{1}\right)$ and $H^{q}\left(Q, G_{2}\right)$ to the group $H^{p+q}(Q, G)$. 
In the case of two-sided operators we also require that $\left(g_{1} \cup g_{2}\right) x$ $=g_{1} x \cup g_{2} x$ and define $f_{1} \cup f_{2}$ by

$$
\begin{aligned}
& \left(f_{1} \cup f_{2}\right)\left(x_{1}, \cdots, x_{p+q}\right) \\
& \quad=f_{1}\left(x_{1}, \cdots, x_{p}\right) x_{p+1} \cdots x_{p+q} \cup x_{1} \cdots x_{p} f_{2}\left(x_{p+1}, \cdots, x_{p+q}\right) .
\end{aligned}
$$

Suppose now that $Q$ is given as a factor group $F / R$ where $F$ is a free group (this amounts to describing $Q$ by generators and relations). More precisely, consider an extension $(F, \psi)$ where $\psi: F \rightarrow Q$ maps $F$ onto $Q$ and has kernel $R$. Let $[R, R]$ denote the commutator group of $R$; then $[R, R]$ is an invariant subgroup of both $R$ and $F$. Define

$$
F_{0}=F /[R, R], \quad R_{0}=R /[R, R] .
$$

The extension $(F, \psi)$ then defines an extension $\left(F_{0}, \psi_{0}\right)$ with $\psi_{0}: F_{0} \rightarrow Q$ mapping $F_{0}$ onto $G$ with the abelian kernel $R_{0}$. According to $\$ 4$ this extension leads to left operators of $Q$ on $R_{0}$ and yields an element $f_{0} \in H^{2}\left(Q, R_{0}\right)$ which describes the extension.

Now consider the group Hom $\left(R_{0}, G\right)$ of all homomorphisms $\phi: R_{0} \rightarrow G$; since $R_{0}=R /[R, R]$ this group may be identified with the group Hom $(R, G)$. Define $Q$ as a group of left operators on Hom $\left(R_{0}, G\right)$ by setting for $x \in Q, \phi: R_{0} \rightarrow G$

$$
(x \phi)(r)=x\left[\phi\left(x^{-1} r\right)\right], \quad r \in R_{0} .
$$

With these operators, the formula

$$
r \cup \phi=\phi(r), \quad r \in R_{0}, \phi \in \operatorname{Hom}\left(R_{0}, G\right),
$$

yields a pairing of the groups $R_{0}$ and Hom $\left(R_{0}, G\right)$ to the group $G$. This in turn leads to a pairing of the cohomology groups $H^{2}\left(Q, R_{0}\right)$ and $H^{q}\left(Q\right.$, Hom $\left.\left(R_{0}, G\right)\right)$ to the group $H^{q+2}(Q, G)$. The cup product reduction theorem (Eilenberg-MacLane [15]) then asserts

(10.1) The homomorphism

$$
\lambda_{q}: H^{q}\left(Q, \operatorname{Hom}\left(R_{0}, G\right)\right) \rightarrow H^{q+2}(Q, G)
$$

defined by

$$
\lambda_{q}(f)=f_{0} \cup f
$$

for $f \in H^{q}\left(Q\right.$, Hom $\left.\left(R_{0}, G\right)\right)$ is an isomorphism onto for $q>0$ and $a$ homomorphism onto for $q=0$.

The kernel of $\lambda_{0}$ can be described as follows. The group $H^{0}\left(Q, \operatorname{Hom}\left(R_{0}, G\right)\right)$ consists of the elements of Hom $\left(R_{0}, G\right)$ which are invariant under the operators of $Q$. This is precisely the group Ophom $\left(R_{0}, G\right)$ of all operator homomorphisms $\phi: R_{0} \rightarrow G$ (that is, homomorphisms satisfying $x(\phi(r))=\phi(x r)$ for all $x \in Q)$. Next define 
$F_{0}$ as a group of left operators on $G$ by setting $a g=\psi_{0}(a) g$ for $a \in F$, $g \in G$. Then every cocycle $f \in Z^{1}\left(F_{0}, G\right)$ when considered on $R_{0}$ yields an element Ophom $\left(R_{0}, G\right)$ while every coboundary $f \in B^{1}(F, G)$ is zero on $R_{0}$. This yields a homomorphism

$$
\eta: H^{1}\left(F_{0}, G\right) \rightarrow \text { Ophom }\left(R_{0}, G\right)=H^{0}\left(Q, \text { Hom }\left(R_{0}, G\right)\right) .
$$

(10.2) The kernel of $\lambda_{0}$ is the image of $\eta$.

For an alternative proof of (10.1) see Lyndon [29].

11. Cohomology groups of cyclic groups. The cup product reduction theorem is particularly adaptable to the computation of the cohomology groups $H^{q}(Q, G)$ in the case of a cyclic group $Q$. Indeed if $Q$ is cyclic of order $h$ then $Q \approx J / h J$ and the group Hom $\left(R_{0}, G\right)$ in the reduction theorem is isomorphic to $G$. This method of computation is adopted in [15]. In view of the frequency with which cyclic groups occur in the applications it may be of interest to have a more direct method for computing the cohomology groups of cyclic groups. The following, hitherto unpublished, discussion has been obtained by the author jointly with S. MacLane.

Let $Q$ be a cyclic group of order $h$ and $\gamma$ a generator of $Q$. Let $G$ be an abelian group with $Q$ as left operators. We set

$$
\begin{aligned}
D g=\gamma g-g, & N g=\sum_{i=0}^{h-1} \gamma^{i} g, \quad \text { for } g \in G, \\
D G=\{\text { all } D g, g \in G\}, & G_{D}=\{\text { all } g \text { with } D g=0\}, \\
N G=\{\text { all } N g, g \in G\}, & G_{N}=\{\text { all } g \text { with } N g=0\} .
\end{aligned}
$$

We verify readily that

$$
N G \subset G_{D}, \quad D G \subset G_{N},
$$

and that these subgroups are independent of the choice of $\gamma$. Clearly $H^{0}(Q, G)=G_{D}$.

For each cochain $f \in C^{q+2}(Q, G)$ define the cochain $\sigma f \in C^{q}(Q, G)$ by setting

$$
(\sigma f)\left(x_{1}, \cdots, x_{q}\right)=\sum_{i=0}^{h-1} \gamma^{-(i+1)} f\left(\gamma, \gamma^{i}, x_{1}, \cdots, x_{q}\right) .
$$

A direct computation shows that

$$
\delta(\sigma f)=\sigma(\delta f)
$$

so that $\sigma$ defines homomorphisms

$$
\sigma: H^{q+2}(Q, G) \rightarrow H^{q}(Q, G),
$$

By iterating $\sigma$ we obtain homomorphisms $(n \geqq 0)$ 


$$
\sigma^{n}: H^{q+2 n}(Q, G) \rightarrow H^{q}(Q, G),
$$

(11.1) Let $Q$ be a cyclic group of order $h$ with generator $\gamma$. Then

$$
H^{2 n+2}(Q, G) \approx G_{D} / N G,
$$

$n \geqq 0$,

where the isomorphism is obtained by assigning to each cocycle $f \in Z^{2 n+2}$ the element $\sigma^{n+1} f$ of $G$. Further

$$
H^{2 n+1}(Q, G) \approx G_{N} / D G, \quad n \geqq 0,
$$

where the isomorphism is obtained by assigning to each cocycle $f \in Z^{2 n+1}$ the element $\left(\sigma^{n} f\right)(\gamma)$ of $G$.

In the argument the following lemma is useful.

(i) If $f \in Z^{q}(Q, G)$ and $f\left(\gamma, x_{2}, \cdots, x_{q}\right)=0$ then $f=0$.

Indeed, developing $(\delta f)\left(\gamma, \gamma^{i}, x_{2}, \cdots, x_{q}\right)$ yields

$$
f\left(\gamma^{i+1}, x_{2}, \cdots, x_{q}\right)=f\left(\gamma^{i}, x_{2}, \cdots, x_{q}\right)
$$

and the proposition follows by induction in $i$.

We shall consider the group of integers $J$ (with $Q$ operating trivially) as paired with $G$ to the group $G$ by setting

$$
m \cup g=m g, \quad m \in J, g \in G .
$$

Further, define a cochain $s_{\gamma} \in C^{2}(Q, J)$ by

$$
\begin{aligned}
& s_{\gamma}\left(\gamma^{i}, \gamma^{j}\right)=0 \text { if } i+j<h, 0 \leqq i, j<h, \\
& s_{\gamma}\left(\gamma^{i}, \gamma^{j}\right)=1 \text { if } i+j \geqq h, 0 \leqq i, j<h .
\end{aligned}
$$

(ii) $s_{\gamma}$ is a cocycle.

This may be shown by a direct computation, using (i) to simplify the argument. Alternatively $s_{\gamma}$ may be realized as the deviation (=factor set) of a group extension $(E, \phi)$ with $E$ the additive group of rational numbers with denominator $h$, and $\phi: E \rightarrow Q$ the homomorphism $\phi(m / h)=\gamma^{m}$. The kernel of this extension is the group $J$. For each $\gamma^{i} \in Q, 0 \leqq i<h$, choose $u\left(\gamma^{i}\right)=i / h$ as representative. Then $u\left(\gamma^{i}\right)+u\left(\gamma^{j}\right)=s_{\gamma}\left(\gamma^{i}, \gamma^{j}\right)+u\left(\gamma^{i+j}\right)$, so that $s$ is indeed the deviation and thus a cocycle. Since $E$ is free this argument also shows that the cohomology class of $s_{\gamma}$ is a cohomology class of the type used in the cup product reduction theorem.

A direct computation shows that

$$
\sigma\left(s_{\gamma} \cup f\right)=f, \quad f \in C^{q}(Q, G) .
$$

Setting for $f \in C^{q+2}(Q, G)$ and $0 \leqq i<h$ 


$$
g\left(\gamma^{i}, x_{2}, \cdots, x_{q-1}\right)=\sum_{j=0}^{i-1} \gamma^{i-j-1} f\left(\gamma, \gamma, x_{2}, \cdots, x_{q-1}\right),
$$

we also find by direct computation (using (i)) that

$$
f+\delta g=s_{\gamma} \cup \sigma f,
$$

Formulae (iii) and (iv) show that for $q>0, \sigma$ maps $H^{q+2}(Q, G)$ isomorphically onto $H^{q}(Q, G)$. The inverse isomorphism is given by the correspondence $f \rightarrow s_{\gamma} \cup f$. This reduces the proof of (11.1) to the cases when $n=0$.

For each $g \in G$ define $g_{\gamma} \in C^{1}(Q, G)$ by

$$
g_{\gamma}\left(\gamma^{i}\right)=\sum_{j=0}^{i-1} \gamma^{i} g, \quad 0 \leqq i<h .
$$

Then, by direct computation (using (i)),

$$
\delta\left(g_{\gamma}\right)=s_{\gamma} \cup N g \text {. }
$$

For each $f \in Z^{1}(Q, G)$ define $g=f(\gamma)$. Since $f\left(\gamma^{i+1}\right)=\gamma f\left(\gamma^{i}\right)+f(\gamma)$ it follows that $g_{\gamma}=f$. Further by (v)

$$
0=\delta f=\delta g_{\gamma}=s_{\gamma} \cup N g
$$

so that $N g=0$ and $g \in G_{N}$. Conversely, $g_{\gamma}(\gamma)=g$ and (v) shows that if $N g=0$ then $g_{\gamma}$ is a cocycle. Thus the correspondence $f \rightarrow f(\gamma)$ yields an isomorphism $Z^{1}(Q, G) \approx G_{N}$. If $f=\delta h$ then $f(\gamma)=\gamma h-h=D h \in D G$ and vice versa. Thus $B^{1}(Q, G)$ corresponds to $D G$. Thus $H^{1}(Q, G)$ is shown to be mapped by $f \rightarrow f(\gamma)$ isomorphically onto $G_{N} / D G$.

For each $f \in Z^{2}(Q, G)$ we have $\sigma f \in Z^{0}(Q, G)=G_{D}$. If $g \in G_{D}$ then $s_{\gamma} \cup_{g} \in Z^{2}(Q, G)$ and by (iii) $\sigma\left(s_{\gamma} \cup g\right)=g$. Thus $\sigma$ maps $Z^{2}$ onto $G_{D}$. If $f=\delta h$ for $h \in C^{1}(Q, G)$ then $\sigma f=\sigma \delta h$ which computes to be $N h(\gamma)$, thus $\sigma f \in N G$. Conversely if $\sigma f \in N g$ then by (v)

$$
s_{\gamma} \cup \sigma f=s_{\gamma} \cup N g=\delta\left(g_{\gamma}\right) .
$$

Since by (iv) $f$ is cohomologous to $s_{\gamma} \cup \sigma f$, it follows that $f$ is a coboundary. Thus $f \in B^{2}(Q, G)$ if and only if $\sigma f \in N G$. Thus $H^{2}(Q, G)$ is shown to be mapped by $f \rightarrow \sigma f$ isomorphically onto $G_{D} / N G$.

This concludes the proof of (11.1). Incidentally we have shown that every cocycle $f \in Z^{2 n+2}(Q, G)$ is cohomologous to a cocycle

$$
s_{\gamma} \cup \ldots \cup s_{\gamma} \cup g
$$

where $g \in G_{D}$ is uniquely determined modulo $N G$; and that every cocycle $f \in Z^{2 n+1}(Q, G)$ is cohomologous to a cocycle 


$$
s_{\gamma} \cup \ldots \cup s_{\gamma} \cup g_{\gamma}
$$

where $g \in G_{N}$ is uniquely determined modulo $D G$.

If $G=J$ is the group of integers with $Q$ as trivial operators the result takes the following form:

(11.2) $H^{2 n+1}(Q, J)=0$. The group $H^{2 n}(Q, J)$ is cyclic of order $h$ and is generated by the cohomology class of the $n$-fold cup product $s^{2 n}=s_{\gamma} \cup \cdots$ $\bigcup_{s_{\gamma}}$. Every cocycle $f \in Z^{2 n}(Q, J)$ is cohomologous to $\sigma^{n}(f) s^{2 n}$. If the generator $\gamma$ of $Q$ is replaced by $\alpha$ where $\gamma=\alpha^{k},(k, h)=1$, then $s_{\alpha}^{2 n}$ is cohomologous to $k^{n} s_{\gamma}^{2 n}$.

The last proposition requires verification. Observe that

$$
\begin{aligned}
\sigma\left(s_{\alpha}\right) & =\sum_{i=0}^{h-1} s_{\alpha}\left(\gamma, \gamma^{i}\right)=\sum_{i=0}^{h-1} s_{\alpha}\left(\alpha^{k}, \alpha^{i k}\right) \\
& =\sum_{i=0}^{h-1} s_{\alpha}\left(\alpha^{k}, \alpha^{i}\right)=\sum_{i=h-k}^{h-1} s\left(\alpha^{k}, \alpha^{i}\right)=k
\end{aligned}
$$

Thus, by (iv), $s_{\alpha}$ is cohomologous $s_{\gamma} \cup k=k s_{\gamma}$.

12. Abelian extensions. We return to the interpretation of the cohomology group $H^{2}(Q, G)$, given in $\$ 4$ as the group $A(Q, G)$ of equivalence classes of group extensions $(E, \phi)$ with $Q$ as quotient and $G$ as kernel and with prescribed operators of $Q$ on $G$. Assume now that $Q$ is abelian and that the operators of $Q$ on $G$ (which is also abelian) are simple. This does not in general imply that $E$ is abelian. In order that $E$ be abelian it is necessary and sufficient that the deviation (or factor set) $f \in Z^{2}(Q, G)$ associated with the extension satisfy the additional symmetry condition

$$
f(x, y)=f(y, x) \text {. }
$$

This leads to the definition of the symmetric subgroup $Z_{s}^{2}(Q, G)$ of $Z^{2}(Q, G)$. Since every coboundary $\delta h \in B^{2}(Q, G)$ is symmetric we obtain a subgroup $H_{s}^{2}(Q, G)$ of $H^{2}(Q, G)$. This subgroup is isomorphic with the subgroup of $A(Q, G)$ given by extensions $(E, \phi)$ with $E$ abelian. Thus $H_{s}^{2}(Q, G)$ may be called the group of abelian group extensions of $Q$ by $G$. It is usually written as $\operatorname{Ext}(Q, G)$.

Suppose now that $Q=F / R$ where $F$ is a free abelian group. Consider the homomorphism

$$
\operatorname{Hom}(F, G) \rightarrow \operatorname{Hom}(R, G)
$$

obtained by restricting each homomorphism of $F$ to the subgroup $R$. Let $\operatorname{Hom}(F, G) \mid R$ denote the image subgroup in $\operatorname{Hom}(R, G)$. Then

$$
\operatorname{Ext}(Q, G) \approx \operatorname{Hom}(R, G) / \operatorname{Hom}(F, G) \mid R \text {. }
$$


This isomorphism is a consequence of the cup product reduction theorem, but can also easily be proved directly. It was proved in Eilenberg-MacLane [11] in connection with the following application to homology theory:

(12.2) In any closure finite abstract complex $K$ the cohomology group $H^{q}(K, G)$ can be expressed by means of the coefficient group $G$ and the integral homology groups $H_{q-1}(K)$ and $H_{q}(K)$ as the direct sum

$$
H^{q}(K, G) \approx \operatorname{Hom}\left(H_{q}(K), G\right)+\operatorname{Ext}\left(H_{q-1}(K), G\right) .
$$

A similar result for homology groups has the form

$$
H_{q}(K, G) \approx H_{q}(K) \otimes G+H_{q-1}(K) * G
$$

where $\otimes$ stands for the tensor product while $*$ denotes a product called the "dual product" that will be discussed in $\$ 13$ ( $\mathrm{H}$. Cartan and Eilenberg [4]).

13. Homology groups of groups. So far our discussion was concerned with the cohomology groups $H^{q}(Q, G)$ and their relations with other concepts of algebra. We now pass to the connections of these groups with topology. For the purpose of this discussion it is useful to have the homology groups $H_{q}(Q, G), G$ discrete abelian. We shall assume throughout this section that $Q$ operates simply on the coefficient groups.

There are at least two methods of introducing these groups. One is based on Pontrjagin's duality theory of abelian groups. Consider a compact abelian group $G$. The group $C^{q}(Q, G)$ of cochains, which is isomorphic to a product of many copies of $G$, can then be topologized in a natural fashion so as to become a compact abelian group. In this way the cohomology group $H^{q}(Q, G)$ becomes a compact abelian group, if $G$ is compact. Given now a discrete abelian group $G$ consider the character group $C h G$ which is compact and define

$$
H_{q}(Q, G)=C h H^{q}(Q, C h G) .
$$

A more direct method of obtaining the homology groups proceeds through the construction of an abstract complex $K(Q)$ associated with the group $Q$. A $q$-dimensional "simplex" of $K(Q)$ is an ordered $q$-tuple $\sigma=\left(x_{1}, \cdots, x_{q}\right)$ of elements of $Q$. For each $0 \leqq i \leqq q$ we define the $i$ th "face" $\sigma^{(i)}$ of $\sigma$ as follows

$$
\begin{aligned}
& \sigma^{(0)}=\left(x_{2}, \cdots, x_{q}\right), \\
& \sigma^{(i)}=\left(x_{1}, \cdots, x_{i} x_{i+1}, \cdots, x_{q}\right), \quad 0<i<q, \\
& \sigma^{(q)}=\left(x_{1}, \cdots, x_{q-1}\right) .
\end{aligned}
$$


This complex $K(Q)$ has sufficiently many properties of a simplicial complex to allow for the usual definition of chains, cochains, homology and cohomology groups. For instance a $q$-cochain with coefficients in $G$ is a function $f$ which to each $q$-simplex $\sigma$ assigns an element $f(\sigma)$ $\in G$. The coboundary $\delta f$ is defined by

$$
(\delta f)(\sigma)=\sum_{i=0}^{q+1}(-1) i f\left(\sigma^{(i)}\right)
$$

for every $(q+1)$-simplex $\sigma$. It thus becomes apparent that the cochains in $K(Q)$ are simply the cochains of $Q$ in $G$. With $Q$ operating simply on $G$, the coboundary operators also coincide so that $H^{q}(K(Q), G)=H^{q}(Q, G)$. Now we can define the homology group $H_{q}(Q, G)$ to be the group $H_{q}(K(Q), G)$ of $K(Q)$.

The second method has the added advantage in that it shows that the various theorems concerning the relations of the various homology and cohomology groups of groups in an abstract complex also hold for the homology and cohomology groups of groups. In particular the isomorphisms

$$
\begin{aligned}
& H^{q}(Q, G) \approx \operatorname{Hom}\left(H_{q}(Q), G\right)+\operatorname{Ext}\left(H_{q-1}(Q), G\right), \\
& H_{q}(Q, G) \approx H_{q}(Q) \otimes G+H_{q-1}(Q) * G
\end{aligned}
$$

hold, where $H_{q}(Q)=H_{q}(Q, J)$ is the integral homology group.

The "Künneth relations" in the cartesian product of two complexes are also applicable to the direct product of two groups and yield an isomorphism (H. Cartan and Eilenberg [4])

$$
H_{r}(Q \times R) \approx \sum_{p+q=r} H_{p}(Q) \otimes H_{q}(R)+\sum_{p+q+1=r} H_{p}(Q) * H_{q}(R) .
$$

Formulae (13.1)-(13.3) allow for a complete computation of the groups $H_{g}(Q, G)$ and $H^{q}(Q, G)$ for $Q$ an abelian group with a finite number of generators and with $Q$ operating trivially on $G$. This was done using a more explicit method by Lyndon [28].

The integral homology group $H_{2}(Q)$ can be given an alternative description. Let $Q=F / R$ where $F$ is a free group. In addition to the commutator group $[F, F]$, consider the group $[F, R]$ spanned by the elements $a r a^{-1} r^{-1}, a \in F, r \in R$. Then

$$
H_{2}(Q) \approx(R \cap[F, F]) /[F, R] .
$$

This isomorphism is an easy consequence of the cup product reduction theorem. Hopf [25] used this isomorphism as a definition of $\mathrm{H}_{2}(Q)$.

An alternative description of the groups $H_{q}(Q, G)$ in the case $G$ is 
a ring with a unit element (and $Q$ operates simply on $G$ ) was given by Hopf [26]; the proof that Hopf's definition agrees with ours can be found in [9].

Assume that $Q$ is abelian. The homology group $H_{1}(Q, G)$ is then symmetric in $Q$ and $G$ and is actually the tensor product $Q \otimes G$. The dual product $Q * G$ may be obtained as follows. Adjoin to the complex $K(Q)$ the symmetry relations

$$
\left(x_{1}, x_{2}\right)=\left(x_{2}, x_{1}\right)
$$

(in dimension two only) and denote the thus modified complex by $K^{\prime}(Q)$. Then $H_{2}\left(K^{\prime}(Q), G\right)$ is symmetric in $Q$ and $G$ and may serve as one of several alternative definitions of the dual product $Q * G(\mathrm{H}$. Cartan and Eilenberg [4]).

14. Relations between the fundamental group and homology groups. Let $X$ be an arcwise connected topological space and $x^{*} \in X$. Relative to $x^{*}$ as base point we define the fundamental group $\pi_{1}=\pi_{1}(X)$ and the higher (abelian) homotopy groups $\pi_{i}=\pi_{i}(X), i>1$. The homology and cohomology groups $H_{q}(X, G)$ and $H^{q}(X, G)$ are defined using singular simplexes, that is, maps $T: s \rightarrow X$ where $s$ is a euclidean simplex with ordered vertices. Since $X$ is arcwise connected, we may without loss of generality assume that $T$ maps all the vertices of $s$ into the point $x^{*}$.

The homotopy group $\pi_{q}(X)$ has a natural homomorphism into the integral homology group $H_{q}(X)$, the image of this homomorphism is denoted by $\Sigma_{q}(X)$ and is called the spherical subgroup of $H_{q}(X)$. The spherical subgroups $\Sigma_{q}(X, G)$ of $H_{q}(X, G)$ can also be defined for an arbitrary coefficient group $G$. In the cohomology group $H^{q}(X, G)$ we distinguish the subgroup $\Lambda^{q}(X, G)$, called the group of spherical annihilators, which is the annihilator of $\Sigma_{q}(X)$ under the natural pairing of $H^{q}(X, G)$ and $H_{q}(X)$ to the group $G$.

Let now $T: s \rightarrow X$ be a singular $q$-simplex where $s$ is a simplex with ordered vertices $v_{0}<\cdots<v_{q}$ all of which are mapped by $T$ into the base point $x^{*}$. The map of the edge $v_{i-1} v_{i}$ into $X$ is then a closed path about $x^{*}$ in $X$ and determines an element $x_{i}$ of the fundamental group $\pi_{1}$. We assign to the singular simplex $T$ the $q$-tuple $\left(x_{1}, \cdots, x_{q}\right)$ of elements of $\pi_{1}$. If $T^{(i)}$ is the $i$ th face of $T$, that is, the face obtained by omitting the vertex $v_{i}$ of $s, i=0, \cdots, q$, then $T^{(i)}$ corresponds respectively to the $(q-1)$-tuples

$$
\begin{array}{cr}
\left(x_{2}, \cdots, x_{q}\right) & \text { if } i=0, \\
\left(x_{1}, \cdots, x_{i} x_{i+1}, \cdots, x_{q}\right) & \text { if } 0<i<q, \\
\left(x_{1}, \cdots, x_{q-1}\right) & \text { if } i=q .
\end{array}
$$


If we compare this with the definition of the complex $K\left(\pi_{1}\right)$ in $\$ 13$ we notice that the correspondence $\kappa: T \rightarrow\left(x_{1}, \cdots, x_{q}\right)$ maps the singular chains of $X$ into chains of $K\left(\pi_{1}\right)$ and that this correspondence commutes with the boundary operator. This leads to homomorphisms

$$
\kappa: H_{q}(X, G) \rightarrow H_{q}\left(\pi_{1}, G\right) .
$$

Similarly we obtain a map for cochains $\kappa: C^{q}\left(\pi_{1}, G\right) \rightarrow C^{q}(X, G)$. Indeed, if $f \in C^{q}\left(\pi_{1}, G\right)$, define

$$
(\kappa f)(T)=f\left(x_{1}, \cdots, x_{q}\right)
$$

where $T \rightarrow\left(x_{1}, \cdots, x_{q}\right)$. This map yields homomorphisms

$$
\kappa: H^{q}\left(\pi_{1}, G\right) \rightarrow H^{q}(X, G) \text {. }
$$

The main result may now be stated

(14.1) In an arcwise connected space $X$ with vanishing homotopy groups $\pi_{i}$ for $1<i<q$ the following isomorphisms hold:

$$
\begin{gathered}
H_{i}(X, G) \approx H_{i}\left(\pi_{1}, G\right), \quad H^{i}(X, G) \approx H^{i}\left(\pi_{1}, G\right) \quad \text { for } i<q, \\
H_{q}(X, G) / \Sigma_{q}(X, G) \approx H_{q}\left(\pi_{1}, G\right), \quad \Lambda^{q}(X, G) \approx H^{q}\left(\pi_{1}, G\right) .
\end{gathered}
$$

This theorem shows the extent to which the group $\pi_{1}$ determines the homology structure of $X$. All the isomorphisms are achieved by the correspondences $\kappa$ described above. The correspondence $\kappa$ for cochains commutes properly with the operation of constructing cup products (in $\pi_{1}$ and in $X$ ). More precisely if $G_{1}$ and $G_{2}$ are paired to the group $G$ and $h_{1} \in H^{i_{1}}\left(\pi_{1}, G_{1}\right), h_{2} \in H^{i_{2}}\left(\pi_{1}, G_{2}\right)$ then

$$
\kappa\left(h_{1} \cup h_{2}\right)=\kappa\left(h_{1}\right) \cup \kappa\left(h_{2}\right) .
$$

Thus for $i_{1}+i_{2} \leqq q$, the group $\pi_{1}$ also determines the cup products in $X$.

In (14.1) the groups $H_{i}\left(\pi_{1}, G\right)$ and $H^{i}\left(\pi_{1}, G\right)$ are understood with $\pi_{1}$ operating trivially on $G$. If we consider homology and cohomology groups with so-called "local coefficients," that is, coefficients $G$ admitting $\pi_{1}$ as a group of operators, the isomorphisms (14.1) remain valid, provided the various groups are suitably interpreted.

As a very special case of (14.1) we obtain the relation

$$
H_{2}(X) / \Sigma_{2}(X) \approx H_{2}\left(\pi_{1}\right) \text {. }
$$

This result (with $X$ a connected simplicial complex and $H_{2}\left(\pi_{1}\right)$ defined by (13.4)) was proved by Hopf [25] in 1942 and was the starting point of this whole investigation. Theorem (14.1) was first published by Eilenberg-MacLane $[12 ; 13]$ and independently and in slightly different forms by Hopf [26], Freudenthal [21], and Eckmann [6]. The isomorphisms (14.1) have been combined with the cup-prod- 
uct reduction theorem to yield some, not yet fully understood, relations between the cohomology groups (with local coefficients) in a simplicial complex (Eilenberg [10]).

15. Generalizations of (14.1). Theorem (14.1) has been extended in several directions.

In the situation of (14.1), the group $\pi_{1}(X)$ determines the factor group $H_{q}(X) / \Sigma_{q}(X)$ of the integral homology group, while the subgroup $\Sigma_{q}(X)$ is a factor group of the $q$ th homotopy group. This leads one to conjecture that perhaps $\pi_{1}, \pi_{q}$ and the operators of $\pi_{1}$ on $\pi_{q}$ jointly determine $H_{q}(X)$ (assuming $\pi_{i}=0$ for $1<i<q$ ). This is not the case as can be shown by examples $[19 ; 34]$. In order to obtain a description of $H_{q}(X)$ in terms of homotopy invariants, a new invariant seems to be necessary. This new invariant (Eilenberg-MacLane [14; 19]) is an element $k^{q+1}(X)$ of the (algebraic) cohomology group $H^{q+1}\left(\pi_{1}, \pi_{q}\right)$, with $\pi_{1}$ operating on $\pi_{q}$, and is defined whenever $\pi_{i}=0$ for $1<i<q$. The invariant $k^{q+1}(X)$ suffices to describe the groups $H_{q}(X, G)$ and $H^{q}(X, G)$ in a purely algebraic manner. The theorem to this effect yields (14.1) as a special case. The definition of $k^{q+1}(X)$ may be roughly described as follows. Consider the function $\kappa$ which maps the singular chains of $X$ into those of $K\left(\pi_{1}\right)$. In order to prove (14.1), an "inverse" function $\bar{\kappa}$ mapping the chains of $K\left(\pi_{1}\right)$ is defined such that $\bar{\kappa}$ commutes with $\partial$ and $\kappa \bar{\kappa}$ is the identity. The definition of $\bar{\kappa}$ utilizes the fact that $\pi_{i}=0$ for $1<i<q$ and can therefore be carried out only up to the dimension $q$ inclusive. The invariant $k^{q+1}(X)$ is obtained by measuring the obstruction against the continuation of $\bar{\kappa}$ to the dimension $q+1$.

The invariant $k^{3}(X) \in H^{3}\left(\pi_{1}, \pi_{2}\right)$ is defined for every (arcwise connected) topological space. Suppose now that $X$ is a simplicial complex containing at least two 2 -simplices. Let $S$ be the 1-dimensional skeleton of $X$ and assume that the base point $x^{*}$ has been chosen in $S$. The group $\pi_{2}(X)$ then maps isomorphically onto the center of the relative homotopy group $\pi_{2}(X, S)$ and there also is a natural homomorphism $\pi_{1}(X) \rightarrow A\left(\pi_{2}(X, S)\right) / I\left(\pi_{2}(X, S)\right.$ ) (see $\left.\$ 5\right)$. Thus $\pi_{2}(X, S)$ may be regarded as a $\pi_{1}(X)$-kernel. The characteristic cohomology class of this kernel is precisely $k^{3}(X)$ (MacLane [32]).

Theorem (14.1) has also been extended by replacing the fundamental group by some higher homotopy group. In this order an abstract complex $K(Q, n)$ is defined for an abelian group $Q$ and an integer $n>1$. The homology and cohomology groups of $K(Q, n)$ over $G$ are denoted by $H_{q}(Q, n, G)$ and $H^{q}(Q, n, G)$. Then (14.1) generalizes as follows:

(15.1) In an arcwise connected topological space $X$ with vanishing 
homotopy groups $\pi_{i}$ for $i<n$ and $n<i<q$ the following isomorphisms hold:

$$
\begin{aligned}
& H_{i}(X, G) \approx H_{i}\left(\pi_{n}, n, G\right), \quad H^{i}(X, G) \approx H^{i}\left(\pi_{n}, n, G\right) \quad \text { for } i<q, \\
& H_{q}(X, G) / \Sigma_{q}(X, G) \approx H_{q}\left(\pi_{n}, n, G\right), \quad \Lambda^{q}(X, G) \approx H^{q}\left(\pi_{n}, n, G\right) .
\end{aligned}
$$

Again in this case an invariant $k_{n}^{q+1}(X)$ belonging to the group $H^{q+1}\left(\pi_{n}, n, \pi_{q}\right)$ is defined and this invariant completely determines the groups $H_{q}(X, G)$ and $H^{q}(X, G)$ (Eilenberg and MacLane [19; 20]).

There are many indications that the study of the groups $H^{q}(Q, n, G)$ will cast light on some of the unsolved problems of homotopy theory. Interesting results have been obtained by $G$. W. Whitehead [35] for the case $q \leqq n+3$, by constructing a space $X$ satisfying the conditions of (15.1) with $\pi_{n}(X) \approx Q$. A purely algebraic study of the groups $H^{q}(Q, n, G)$, falling back on the algebraic complex $K(Q, n)$, has been undertaken by the author and S. MacLane, and is in progress. So far, complete results were obtained only for $q \leqq n-2$.

Extensions of (14.1) and (15.1) to the case of relative homotopy and homology groups have been made by Blakers [3].

16. Equivariant groups of complexes with operators. The constructions leading to the proof of (14.1) were carried out in the space $X$. Instead one might carry out these constructions in the universal covering space $\tilde{X}$ of $X$ and treat $\pi_{1}(X)$ as a group of homeomorphisms operating on $\tilde{X}$. One is thus lead to the study of complexes and spaces with a group of operators (Eckmann [6], Eilenberg [9]) which furnishes generalizations of (14.1) in a different direction.

Let $K$ be an abstract complex. We shall say that $Q$ operates on $K$ if, for every $q$, the group $Q$ operates (on the left) on the group of $q$ chains $C_{q}(K)$ in such fashion that the boundary operator $\partial: C_{q}(K)$ $\rightarrow C_{q-1}(K)$ is an operator homomorphism. Let $G$ be an abelian coefficient group with $Q$ as a group of (left) operators. A $q$-cochain $f$ of $K$ over $G$, that is, a homomorphism $f: C_{q}(K) \rightarrow G$, will be called equivariant provided

$$
f(x c)=x f(c), \quad x \in Q, c \in C_{g}(K) .
$$

The coboundary of an equivariant is equivariant and therefore using equivariant cochains throughout we arrive at an equivariant cohomology group $H_{e}^{q}(K, G)$. This group admits a natural homomorphism

$$
H_{e}^{q}(K, G) \rightarrow H^{q}(K, G)
$$


into the ordinary cohomology group of $K$.

The complex $K$ is called acyclic in dimensions less than $q$ if the integer homology groups $H_{i}(K)$ vanish for $i<q$, with $H_{0}(K)$ interpreted as the reduced homology group. We shall say that $Q$ operates on $K$ freely provided for each $q \geqq 0$, the group $C_{q}(K)$ contains a set $\Omega_{q}$ such that the elements $x \omega\left(x \in Q, \omega \in \Omega_{q}\right)$ form a free base for the abelian group $C_{q}(K)$. It can be shown quite easily that if $K$ is acyclic in dimensions less than $q$ and $Q$ operates freely on $K$, then the equivariant cohomology groups $H_{e}^{i}(K, G), i<q$, are completely determined by $Q, G$ and the operators of $Q$ on $G$. In order to find precisely what these groups are, associate with the group $Q$ an abstract complex $K_{Q}$ as follows:

A $q$-dimensional "simplex" of $K_{Q}$ is an ordered $(q+1)$-tuple $\sigma=\left(x_{0}, \cdots, x_{q}\right)$ of elements of $Q$. The $i$ th face of $\sigma(0 \leqq i \leqq q)$ is $\sigma^{(i)}=\left(x_{0}, \cdots, \hat{x}_{i}, \cdots, x_{q}\right)$, where the circumflex indicates that $x_{i}$ is omitted. The operators of $Q$ on $K_{Q}$ are given by $x\left(x_{0}, \cdots, x_{q}\right)$ $=\left(x x_{0}, \cdots, x x_{q}\right)$. The complex $K$ is acyclic in all dimensions and $Q$ operates freely on $K$. A $q$-dimensional cochain of $K$ over $G$ is simply a function $f$ of $q+1$ variables in $Q$ with values in $G$. The coboundary $\delta f$ is defined by

$$
\delta f\left(x_{0}, \cdots, x_{q+1}\right)=\sum_{i=0}^{q+1}(-1)^{i} f\left(x_{0}, \cdots, \hat{x}_{i}, \cdots, x_{q+1}\right)
$$

while the equivariance condition is

$$
f\left(x x_{0}, \cdots, x x_{q}\right)=x f\left(x_{0}, \cdots, x_{q}\right) .
$$

With each such equivariant cochain $f$ we associate a cochain $\tilde{f} \in C^{q}(Q, G)$ by setting

$$
\bar{f}\left(x_{1}, \cdots, x_{q}\right)=f\left(1, x_{1}, x_{1} x_{2}, \cdots, x_{1} x_{2} \cdots x_{q}\right) .
$$

Conversely $\bar{f}$ defines $f$ by

$$
f\left(x_{0}, \cdots, x_{q}\right)=x_{0} \bar{f}\left(x_{0}^{-1} x_{1}, \cdots, x_{q-1}^{-1} x_{q}\right) .
$$

Moreover $\delta \bar{f}=\overline{\delta f}$. Thus the equivariant cohomology groups $H_{e}^{q}\left(K_{Q}, G\right)$ simply yield an alternative definition of the cohomology groups $H^{q}(Q, G)$. We refer to this definition as the "homogenous definition" in contrast with the original "nonhomogenous definition". If in the complex $K_{Q}$ we introduce the relations $x\left(x_{0}, \cdots, x_{q}\right)=\left(x x_{0}, \cdots, x x_{q}\right)$ we obtain a complex isomorphic with $K(Q)$ as defined in $\$ 13$. Thus $K_{Q}$ may be regarded as a "univeral covering" of $K(Q)$.

The precise theorem is then the following.

(16.1) Let $K$ be a complex acyclic in dimensions less than $q$ with the 
group $Q$ operating freely on $K$. For every abelian coefficient group $G$ with $Q$ as (left) operators, the following isomorphisms hold:

$$
\begin{aligned}
& H^{i}(Q, G) \approx H_{\theta}^{i}(K, G) \\
& \text { for } i<q \text {, } \\
& H^{q}(Q, G) \approx \text { kernel of } H_{e}^{q}(K, G) \rightarrow H^{q}(K, G) \text {. }
\end{aligned}
$$

In particular if $Q$ operates without fixed points on a topological space $X$, acyclic in dimensions less than $q$, then $Q$ operates freely on the singular complex $S(X)$ of $X$ and (16.1) can be applied.

In order to derive (14.1) (for cohomology only) from (16.1) the following facts are needed: $\left(1^{\circ}\right) \pi_{1}(X)$ operates on the universal covering space $\tilde{X}$ without fixed points, $\left(2^{\circ}\right)$ the condition $\pi_{i}(X)=0$ for $1<i<q$ is equivalent with the acyclicity of $\tilde{X}$ in dimensions less than $q,\left(3^{\circ}\right)$ under the natural projection $\tilde{X} \rightarrow X$, the group $H_{e}^{b}(\tilde{X}, G)$ maps isomorphically onto the group $H^{i}(X, G)$, to be taken with local coefficients if $\pi_{1}(X)$ operates on $G,\left(4^{\circ}\right)$ if $\pi_{i}(X)=0$ for $1<i<q$ then the kernel of $H_{e}^{q}(\tilde{X}, G) \rightarrow H^{q}(\tilde{X}, G)$ is mapped isomorphically onto the subgroup $\Lambda^{q}(X, G)$ of $H^{q}(X, G)$.

In addition to the ordinary and equivariant cohomology group $H^{q}(K, G)$ and $H_{e}^{q}(K, G)$ we may introduce a residual cohomology group $H_{r}^{q}(K, G)$ which is obtained by calculating modulo equivariant cochains. More precisely $H_{r}^{q}=Z_{r}^{q} / B_{r}^{q}$ where $f \in Z_{r}^{q}$ if and only if $\delta f$ is equivariant, while $f \in B_{r}^{q}$ if and only if $f=\delta g+h$ with $h$ equivariant. The three kinds of cohomogy groups are connected by homomorphisms

$$
\cdots \rightarrow H_{e}^{q}(K, G) \rightarrow H^{q}(K, G) \rightarrow H_{r}^{q}(K, G) \rightarrow H_{e}^{q+1}(K, G) \rightarrow \cdots
$$

and form an exact sequence in the sense that the image of each homomorphism is the kernel of the next one.

In the residual group $H_{r}^{q}(K, G)$ a subgroup $D_{r}^{q}(K, G)$ is distinguished by considering cochains $f \in C^{q}(K, G)$ such that $f(x z)=x f(z)$ for any $x \in Q$ and $z \in C_{q}(K)$ with $\partial z=0$. [9]):

Proposition (16.1) may then be supplemented as follows (Eilenberg

(16.2) Under the conditions of (16.1) the following isomorphisms hold:

$$
\begin{aligned}
& H^{i+1}(Q, G) \approx H_{r}^{i}(K, G) \\
& H^{q+1}(Q, G) \approx D_{r}^{q}(K, G) .
\end{aligned}
$$$$
\text { for } i<q \text {, }
$$

This last isomorphism, expressed in the dual language of homology, leads to the following result due to Hopf [27].

(16.3) Let $X$ be a q-dimensional connected polyhedron with $\pi_{i}(X)=0$ 
for $1<i<q$. Denote by $\gamma_{q}$ the kernel of the natural homomorphism $\pi_{q}(X)$ $\rightarrow H_{q}(X)$, and by $\pi_{q, 0}$ the subgroup of $\pi_{q}$ spanned by all elements $\alpha-x \alpha$ with $\alpha \in \pi_{q}, x \in \pi_{1}$. Then $\pi_{q, 0} \subset \gamma$ and

$$
H_{q+1}\left(\pi_{1}\right) \approx \gamma_{q} / \pi_{q, 0}
$$

An analogous discussion of equivariant and residual homology groups is possible without interesting variations.

17. Generalizations. In analogy to the invariant $k^{q+1}(X)$ $\in H^{q+1}\left(\pi_{1}, \pi_{q}\right)$ indicated in $\$ 15$, an invariant $l^{q+1}(K) \in H^{q+1}\left(Q, H_{q}(K)\right)$ may be defined for every complex $K$ acyclic in dimensions less than $q$ with $Q$ as a group of operators. It was shown by the author and $\mathrm{S}$. MacLane [19] that this invariant $l^{q+1}(K)$ determines the group $H_{e}^{q}(K, G)$ in its entirety as well as the homomorphism $H_{e}^{q}(K, G)$ $\rightarrow H^{q}(K, G)$. Further it was proved by the author (unpublished) that $l^{a+1}(K)$ determines also the group $H_{r}^{q}(K, G)$ and the homomorphism $H^{q}(K, G) \rightarrow H_{r}^{q}(K, G)$. This implies that $l^{q+1}(K)$ also determines the kernel of $H_{e}^{q+1}(K, G) \rightarrow H^{q+1}(K, G)$.

Applying these results to the universal covering $\tilde{X}$ of a space $X$ leads to the following result, supplementing (14.1).

(17.1) Let $X$ be an arcwise connected space with vanishing homotopy groups $\pi_{i}$ for $1<i<q$. The invariant $k^{q+1}(X) \in H^{q+1}\left(\pi_{1}, \pi_{q}\right)$ then determines the homology groups $H_{q}(X, G)$ as well as the factor group of $H_{q+1}(X, G)$ by the image of $H_{q+1}(\tilde{X}, G)$ under the natural projection of the universal convering $\tilde{X}$ into $X$. For integer coefficients $k^{q+1}(X)$ determines $H_{q+1}(X) / \Sigma_{q+1}(X)$. For cohomology $k^{q+1}(X)$ determines $H^{q}(X, G)$ and the kernel of $H^{q+1}(X, G) \rightarrow H^{q+1}(\tilde{X}, G)$.

An interesting generalization of the equivariant theory has been recently introduced by Eckmann $[7 ; 8]$. Let $K$ be a complex with $Q$ as a group of operators and let $G$ be any abelian coefficient group (not necessarily with $Q$ as operators). Let $\psi$ be any subgroup of the group of all functions $Q \rightarrow G$. A cochain $f \in C^{q}(K, G)$ is called $\psi$-cochain if for every $c \in C_{q}(K)$ the expression $f(x c)$ treated as function of $x \in Q$ belongs to $\psi$. Replacing the equivariant cochains by $\psi$-cochains the $\psi$-groups $H_{\psi}^{q}(K, G)$ and the corresponding residual groups are defined.

In order to obtain the equivariant theory assume that $Q$ operates on $G$ and take $\psi$ to be the group of all functions $x \rightarrow x g, g \in G$.

The next important case is the "almost zero" theory where $\psi$ is taken to be the group of all functions $Q \rightarrow G$ which are zero except for a finite number of elements of $Q$. This choice is especially important if $K$ is a locally finite simplicial (or cellular) complex which has a finite fundamental domain relative to the operators of $Q$. In this case the $\psi$-cochains are the finite cochains of $Q$ so that $H_{\psi}^{q}(Q, G)$ is the coho- 
mology group of $K$ based on finite cochains. It is independent of the group $Q$.

The group $H_{\psi}^{q}\left(K_{Q}, G\right)$ in the "almost zero" theory is denoted by $\Pi^{q}(Q, G)$. More directly, consider as $q$-cochains, functions $f\left(x_{0}, \cdots, x_{q}\right)$ $\in G$, defined for $x_{0}, \cdots, x_{q} \in Q$ such that for each system $x_{0}, \cdots, x_{q}$

$$
f\left(x x_{0}, \cdots, x x_{q}\right)=0
$$

for all, except for a finite number, of $x \in Q$. Define the coboundary $\delta f$ as

$$
(\delta f)\left(x_{0}, \cdots, x_{q+1}\right)=\sum_{i=0}^{q+1}(-1) i f\left(x_{0}, \cdots, \hat{x}_{i}, \cdots, x_{q+1}\right) .
$$

Then $\Pi^{q}(Q, G)$ may be defined using the usual procedure for defining homology and cohomology groups.

All the results discussed in $\$ 16$ remain valid for any choice of $\psi$. The same holds for the results involving an invariant $l^{q+1}(K)$. Curiously enough, this invariant remains in the group $H^{q+1}(Q, G)$, which is the equivariant group of $K_{Q}$. This shows that the equivariant theory has some features of universality.

Using the "almost zero" theory Eckmann has proved the following theorem due to Specker (unpublished).

(17.2) In an orientable 3-dimensional manifold the fundamental group $\pi_{1}$ determines the homotopy group $\pi_{2}$ :

$$
\pi_{2} \approx \Pi^{1}\left(\pi_{1}, J\right)
$$

where $J$ is the additive group of integers.

\section{BIBLIOGRAPHY}

1. E. Artin, C. J. Nesbitt and R. M. Thrall, Rings with minimum conditions, University of Michigan Press, Ann Arbor, 1944.

2. R. Baer, Automorphismen von Erweiterungsgruppen, Actualités scientifiques et Industrielles, No. 205, Paris, 1935.

3. A. L. Blakers, Some relations between homology and homotopy groups, Ann. of Math. vol. 49 (1948) pp. 428-461.

4. H. Cartan and S. Eilenberg, Products of groups and complexes (in preparation).

5. C. Chevalley and S. Eilenberg, Cohomology theory of Lie groups and Lie algebras, Trans. Amer. Math. Soc. vol. 63 (1948) pp. 85-124.

6. B. Eckmann, Der Cohomologie-Ring einer beliebigen Gruppe, Comment. Math. Helv. vol. 18 (1945-1946) pp. 232-282.

7. - On complexes over a ring and restricted cohomology groups, Proc. Nat. Acad. Sci. U.S.A. vol. 33 (1947) pp. 275-281.

8. - On infinite complexes with automorphisms, Proc. Nat. Acad. Sci. U.S.A. vol. 33 (1947) pp. 372-376. 
9. S. Eilenberg, Homology of spaces with operators. I, Trans. Amer. Math. Soc. vol. 61 (1947) pp. 378-417; Errata vol. 62 (1947) p. 548.

10. - Relations between cohomology groups in a complex, Comment. Math. Helv. vol. 21 (1948) pp. 302-320.

11. S. Eilenberg and S. MacLane, Group extensions and homology, Ann. of Math. vol. 43 (1942) pp. 757-831.

12. - Relations between homology and homotopy groups, Proc. Nat. Acad. Sci. U.S.A. vol. 29 (1943) pp. 155-158.

13. - Relations between homology and homotopy groups of spaces, Ann. of Math. vol. 46 (1945) pp. 480-509.

14. - Determination of the second homology and cohomology groups of a space by means of homotopy invariants, Proc. Nat. Acad. Sci. U.S.A. vol. 32 (1946) pp. 277280.

15. - Cohomology theory in abstract groups. I, Ann. of Math. vol. 48 (1947) pp. 51-78.

16. - Cohomology theory in abstract groups. II, Group extensions with a nonAbelian kernel, Ann. of Math. vol. 48 (1947) pp. 326-341.

17. - Algebraic cohomology groups and loops, Duke Math. J. vol. 14 (1947) pp. $435-463$.

18. - Cohomology and Galois Theory. I. Normality of algebras and Teichmilller's cocycle, Trans. Amer. Math. Soc. vol. 64 (1948) pp. 1-20.

19. - Homology of spaces with operators. II, Trans. Amer. Math. Soc. vol. 65 (1949).

20. - Relations between homology and homotopy groups of spaces. II, Ann. of Math. (in print).

21. H. Freudenthal, Der Einfluss der Fundamentalgruppe auf die Bettische Gruppen, Ann. of Math. vol. 47 (1946) pp. 274-316.

22. G. Hochschild, On the cohomology groups of an associative algebra, Ann. of Math. vol. 46 (1945) pp. 58-67.

23. - On the cohomology theory for associative algebras, Ann. of Math. vol. 47 (1946) pp. 568-579.

24. - Cohomology and representations of associative algebras, Duke Math. J. vol. 14 (1947) pp. 921-948.

25. H. Hopf, Fundamentalgruppe und zweite Bettische Gruppe, Comment. Math. Helv. vol. 14 (1942) pp. 257-309; Nachtrag zu ... vol. 15 (1943) pp. 27-32.

26. - Ueber die Bettische Gruppen die zu einer beliebigen Gruppe gehören, Comment. Math. Helv. vol. 17 (1945) pp. 39-79.

27. —- Beiträge zur Homotopietheorie, Comment. Math. Helv. vol. 17 (1945) pp. 307-333.

28. R. G. Lyndon, The cohomology theory of group extensions, Duke Math. J. vol. 15 (1948) pp. 271-292.

29. - New proof for a theorem of Eilenberg and MacLane, Ann. of Math. (in print).

30. S. MacLane, Symmetry of algebras over a number field, Bull. Amer. Math. Soc. vol. 54 (1948) pp. 328-333.

31. - A non-associative method for associative algebras, Bull. Amer. Math. Soc. vol. 54 (1948) pp. 897-902.

32. - Cohomology theory in abstract groups. III, Operator homomorphisms of kernels, Ann. of Math. (in print). 
33. O. Teichmuller, Ueber die sogenannte nichtkommutative Galoissche Theorie und die Relation $\xi_{\lambda, \mu, \nu} \xi_{\lambda, \mu \nu, \pi} \xi^{\lambda}, \nu, \pi=\xi_{\lambda, \mu, \nu \pi} \xi_{\lambda \mu, \nu, \pi}$, Deutsche Mathematik vol. 5 (1940) pp. 138149.

34. H. S. Wang, Some examples concerning the relations between homology and homotopy groups, K. Nederlandsche Akademie van Wetenschappen, Proceedings vol. 50 (1947) pp. 873-875 = Indagationes Mathematical vol. 9 (1947) pp. 384-386.

35. G. W. Whitehead, On spaces with vanishing low-dimensional homotopy groups, Proc. Nat. Acad. Sci. U. S. A. vol. 34 (1948) pp. 207-211.

Columbia University 